\title{
The mechanics of decompressive craniectomy: Bulging in idealized geometries
}

\author{
Johannes Weickenmeier ${ }^{\ddagger}$, Ellen Kuhl ${ }^{\ddagger}$, and Alain Goriely* \\ ${ }^{\ddagger}$ Mechanical Engineering, Stanford University \\ * Mathematical Institute, University of Oxford \\ Corresponding author: goriely@maths.ox.ac.uk
}

April 29, 2016

\begin{abstract}
In extreme cases of traumatic brain injury or a stroke, the resulting uncontrollable swelling of the brain may lead to a harmful increase of the intracranial pressure. As a common measure for immediate release of pressure on the brain, part of the skull is surgically removed allowing for the brain to bulge outwards, a procedure known as a decompressive craniectomy. During this excessive brain swelling, the affected tissue typically undergoes large deformations resulting in a complex three-dimensional mechanical loading state with several important implications on optimal treatment strategies and outcome. Here, as a first step towards a better understanding of the mechanics of a decompressive craniectomy, we consider simple models for the bulging of elastic solids under geometric constraints representative of the surgical intervention. In small deformations and simple geometries, the exact solution of this problem is derived from the theory of contact mechanics. The analysis of these solutions reveals a number of interesting generic features relevant for the mechanics of craniectomy.
\end{abstract}

\section{Introduction}

Brain swelling can be caused by a number of factors, including haemorrhages, infection, tumour growth, high altitude, ischemic strokes, or traumatic brain injury [1]. In particular, severe traumatic brain injury and malignant middle cerebral artery infarctions can result in a critical increase of intracranial pressure strongly associated with a compromised regulation of cerebral blood flow and severe consequences on patient health [2]. During swelling or increased intracranial pressure, the corresponding tissue compression may lead to a further impairment of surrounding blood vessels. This load restricts blood flow and, without immediate treatment, leads to an expanding ischemic zone and non-recoverable damage to the brain [3]. Especially swelling and ischemia bear the danger of propagation through the brain unless properly treated medically [4]. The management of elevated intracranial pressure 
(defined usually as more than $20 \mathrm{mmHg}$ within any intracranial space) is therefore critical to prevent severe brain damage or death.

Since swelling is caused by an osmotic imbalance in which capillary fluid perfuses into the tissue, the primary non-invasive treatment for cerebral edema and elevated intracranial pressure is osmotherapy [5]. In osmotherapy, substances such as mannitol or hypertonic saline are administered intravenously to increase serum osmolality [6]. While osmotherapy can effectively decrease the acutely elevated intracranial pressure in some situations, its long-term effects may be limited [7]. For instance, cytotoxic edema creates an imbalance at the cellular level that is resistant to known treatments [8]. Similarly, if the integrity of the blood brain barrier is compromised, osmotically active agents will penetrate the tissue and fail to establish an osmotic gradient. Other treatments include hyperventilation, ventricular drainage, induced coma, and hypothermia [9].

\section{$1.1 \quad$ Description}

When medical management of elevated intracranial pressure fails, a decompressive craniectomy serves as a measure of last resort in order to ensure an immediate release of intracranial pressure and to allow for the compressed and potentially ischemic brain tissue to expand outward. Craniectomy is used both to prevent further swelling and to decrease intracranial pressure [10]. This highly invasive procedure takes place with the following steps [11, 12]: (i) scalp incision, (ii) removal of the bone flap (a large portion of the skull), (iii) opening of the dura mater (the thick outermost membrane covering the brain), (iv) closure of the skin incision. Only after a full resorption of the swelling, within days to weeks, a cranioplasty or cranial bone replacement is performed in order to protect the exposed brain tissue. Fig. 1 shows the potential extent of skull bone removal for an exemplary but severe decompressive craniectomy.
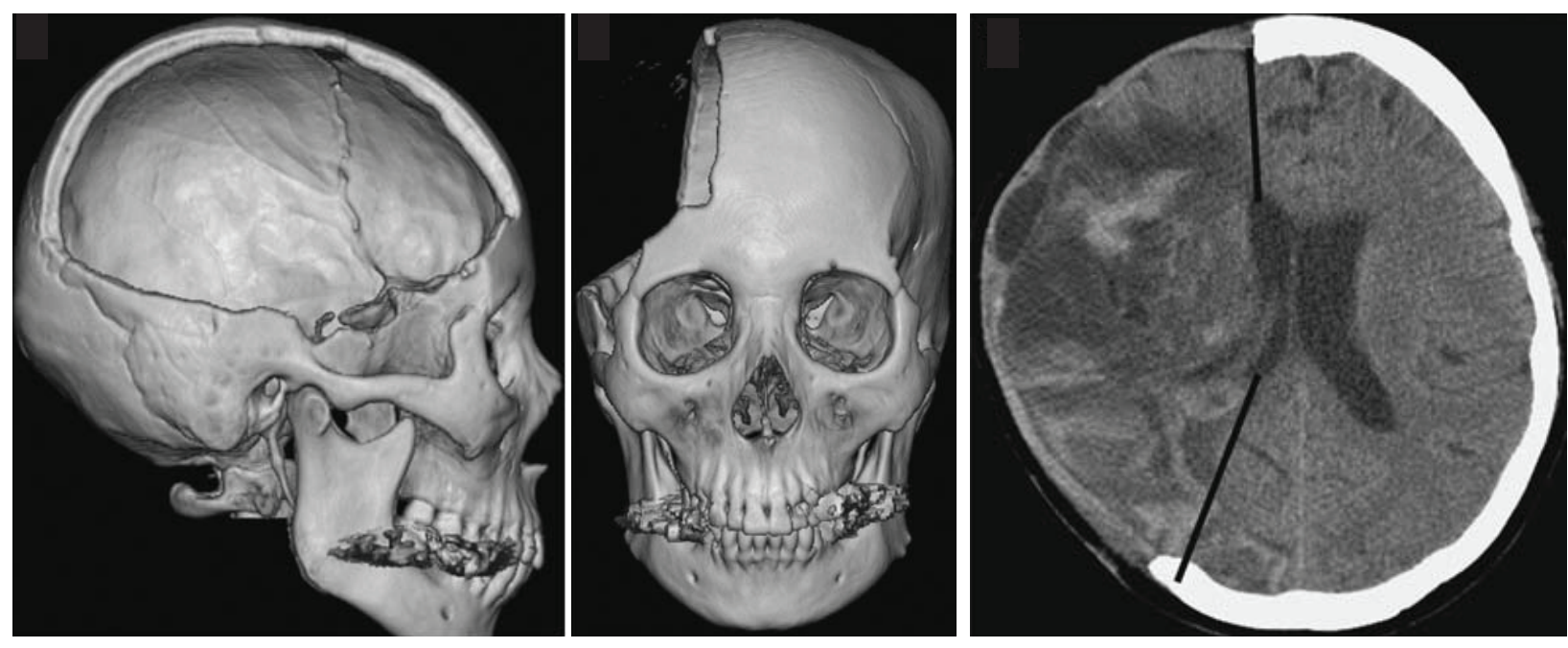

Figure 1: Large decompressive craniectomy on a patient with malignant middle cerebral artery infarction. Left: lateral view; middle: anterior view; right: transverse view. The axial section of the computed tomography scan shows the approximated middle cerebral artery territory. (Reproduced from [13] without permission). 


\subsection{A short history}

Trephination, the surgical intervention in which holes are drilled in the skull, has been performed since prehistoric times and was first described by Hippocrates for the treatment of severe head injuries. However, the procedure of removing a large part of the skull for decompression was first described by Annandale in 1894, and proposed as a means to relieve intracranial pressure following traumatic brain injury by Kocher in 1901 [14] and for brain tumors by Cushing in 1905 [15]. Decompressive craniectomy gained popularity in the late 1960's and early 1970's when encouraging results in the treatment of malignant brain oedema were published [16]. However, soon after, further studies showed conflicting results and poor outcome. For decades, the procedure was known as potentially life-saving but associated with serious complications and was eventually broadly abandoned. The surgical procedure commonly applied today was introduced in 1999 [17] for the treatment of severe traumatic brain injury. However, despite numerous examples of successful treatment outcomes, the medical efficacy of the decompressive craniectomy remains controversial.

\subsection{The craniectomy dilemma}

In a landmark 2011 paper, Cooper et al. [18] considered a group of patients with refractory intracranial hypertension ( defined as an intracranial pressure higher than $20 \mathrm{~mm} \mathrm{Hg}$ for more than 15 minutes despite medical therapy). All subjects were randomly assigned to a group where they receive bi-frontal temporoparietal decompressive craniectomy or to a non-surgical group where they receive standard care only. The study showed a significant decrease in intracranial pressure in patients in the surgical group. However, long-term clinical outcomes were worse for subjects who underwent surgery, thus raising questions about the benefits of a craniectomy. It should be noted here, however, that the conclusions presented by [18], received viable criticism $[19,20]$.

Another study found that decompressive craniectomy for stroke patients increases the changes of survival in comparison to other medical treatments [12]. At the same time, the authors stress the imminent possibility of irreparable disability and strongly forewarn the indiscriminate use of this highly invasive surgical intervention. Nevertheless, decompressive craniectomy remains an effective treatment method in refractory intracranial hypertension, especially when the corresponding hypertension is expected to result in excessive ischemic cell death, tissue necrosis, or even death. Most current reviews and studies related to craniectomy conclude that further studies and reviews are necessary to develop strong medical indicators for the necessity and benefit of this procedure [21, 22, 12, 23].

The main craniectomy dilemma faced by neurosurgeons can be understood in simple mechanical terms: On the one hand, an increase in stress due to elevated intracranial pressure leads to ischemia that is likely to propagate and destroy large areas of the brain. On the other hand, a surgically induced release of stresses comes at the cost of critically large strains. In particular, it has been shown that axonal strains as low as 3-5\% can create internal axonal damage [24], and strains higher than $20 \%$ can induce significant levels of cell injury, electrophysiological changes, and morphological damage in the white matter $[25,26]$. The massive outward swelling upon skull bone removal creates zones of high stretch [27] which, in turn, can create long-term axonal damage and compromise clinical efficacy [18]. The craniectomy dilemma may therefore be phrased as a mechanical problem of stresses versus 
strains. It is crucial to have a full theoretical understanding of the underlying deformation as well as the stresses generated by this process. Our primary objective in the present work is to provide a thorough theoretical description of soft elastic tissue swelling-induced herniation and a derivation of the associated stress field.

Most of the published literature focuses on the statistical treatment of patient data in randomized studies or on meta-analyses. The basic aspects of deformation in simplified spherical geometries have been used as guidelines to determine the size of the bone flap necessary to relieve a certain volume of edema. This simple computation is reviewed in Section 2.

The finite-element method is a natural approach to compute soft-tissue deformation. Different groups have used these computational methods together with image registration to predict the intraoperative brain shift during craniotomy and, in particular, to follow the midline shift, a critical indicator of tissue deformations used by neurosurgeons [28, 29, 30, 31].

Decompressive craniectomy usually leads to significantly large deformations and it remains to be verified that the small-strain theory remains valid when observing large tissue deformations. In particular, the choice of the material model and the numerical solver are highly dependent on the loading situation and stress state and play a crucial role in computational mechanics [32]. Nevertheless, the simulation of craniectomy has been investigated by different groups in recent years. Amongst these, an interdisciplinary research group in Stockholm combined finite-element models with medical imaging (computerized tomography (CT), magnetic resonance imaging (MRI), and positron emission tomography (PET)) to obtain parameters such as intracranial pressure, strain, and stress [33]. For craniectomy, these computational methods allow to extract axonal stretches directly from CT scans [34, 27]. Some computational simulations also suggest that if a decompressive craniectomy is performed at the opposite side of the injury, it has the potential to improve patient outcome [35]. In an excellent study, Fletcher et al. developed CT image-based methods to quantify brain deformation following a decompressive craniectomy [36]. In particular, they used the experimentally observed deformation behavior of constrained swelling gels to validate their method [37].

In the present work, we study the mechanics of decompressive craniectomy in idealized conditions of increasing complexity to gain insight into the basic trade-off between stress and strain and the balance between skull opening and the generation of high contact stress and tissue stretch. We believe that a systematic theoretical study may provide useful insight for the development of advanced numerical methods and could potentially serve as a guide for surgical planning.

\section{Geometric estimates}

The first step in studying craniectomy is to provide a geometric characterization of volume change due to swelling. If the bone flap is of a certain size, how big would the bulge be for a given amount of additional swollen volume [38, 13]? Or, conversely, how big should the skull opening be for a given swollen volume $[39,40]$ ? Surprisingly, these simple questions have not been adequately addressed in the literature so far. Estimates are either mathematically dubious or their description is so baffling and incomplete that they can neither be understood 
nor reproduced. It is a testament of the great mutual respect that medical scientists have for each other that the curves produced by such mysterious processes found their way into publications.

To obtain geometric estimates, we have to make assumptions about the final shape of the bulge. Different authors have assumed that the shape is either spherical [41] or cylindrical [39], or they simply looked at the projected surface [36]. In this last study, Fletcher et al. performed a careful analysis of area and volume prior to and after swelling.

Simple estimates can be obtained by assuming a spherical shape for skull, brain, and the bulge while the tissue is considered incompressible. We recall that the volume $V$ and surface area $A$, as shown in Fig. 2, are given by

$$
V(R, h)=\frac{\pi h}{6}\left(3 a^{2}+h^{2}\right), \quad A(R, h)=2 \pi R h,
$$

where $R$ is the radius of the sphere, $2 a$ is the cap opening, and $h$ the height. The different variables introduced in Fig. 2 are related by

$$
\begin{aligned}
& a^{2}=2 h R-h^{2}, \\
& a=R \sin \theta, \\
& y=R \cos \theta, \\
& h=R-y=R(1-\cos \theta),
\end{aligned}
$$

where $\theta$ is the opening angle of the cap. Upon swelling, the shape of the emerging bulge is
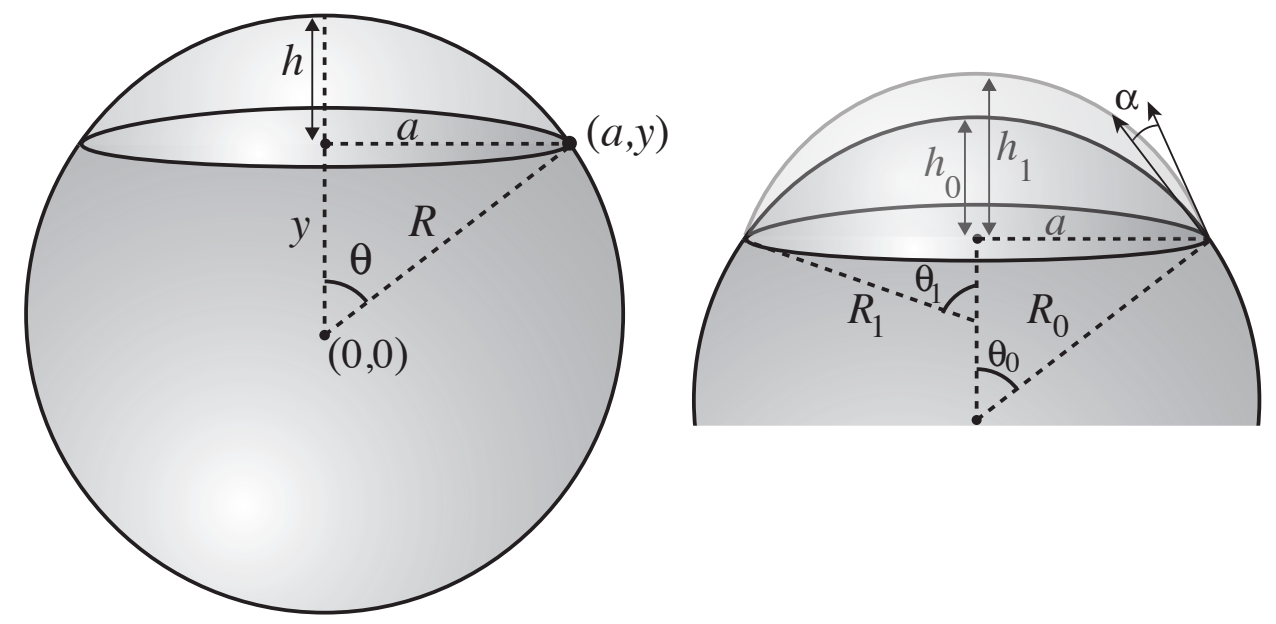

Figure 2: Left: Basic geometric variables defining a spherical cap in a sphere of radius $R$. Right: Idealized deformation in response to craniectomy, assuming that the bulge is also spherical; $h_{0}$ is the initial height and $h_{1}$ the height of the deformed tissue.

modeled as a sphere as well and the volume added due to swelling is defined by the difference in volume of both caps:

$$
\Delta V=V\left(R_{1}, h_{1}\right)-V\left(R_{0}, h_{0}\right)=\frac{\pi}{6}\left[3 a^{2}\left(h_{1}-h_{0}\right)+\left(h_{1}^{3}-h_{0}^{3}\right)\right] .
$$


Due to a high inter-subject variability in brain volume, we define a relative measure for the volume change as follows

$$
\delta=\frac{\Delta V}{V_{0}}=\frac{3}{4 \pi R_{0}^{3}}\left[V\left(R_{1}, h_{1}\right)-V\left(R_{0}, h_{0}\right)\right] .
$$

Note that, since the opening is shared by the two spheres, we have the following relationship between the two radii:

$$
a=R_{0} \sin \theta_{0}=R_{1} \sin \theta_{1} .
$$

A simple problem that the surgeon faces is to determine the dimensions of the skull opening in terms of the opening radius $a$ or opening angle $\theta_{0}$, respectively, which can accommodate the desired volume increase $\delta$. Assuming that $\theta_{0}$ and $\delta$ in $(7)$ are fixed, we see that a desired volume increase may be realized by a range of opening sizes $a$ by varying $\theta_{1}$. To determine a unique solution, we require an additional constraint. One such constraint is given by the bulging angle $\alpha$, defined by the tangents to both spheres at their intersection point, as indicated in Fig. 2. If this angle is too high, we expect high stress at the point of contact and possible herniation, a medically highly unfavorable state of the tissue. The bulging angle is given by $\alpha=\theta_{1}-\theta_{0}$ and is prescribed to reduce tissue loading at the edge of the bulge. Consequently, the added volume $\delta$ reduces to a function of bulge angle $\alpha$ and opening angle $\theta_{0}$ and takes the form

$$
\delta=\frac{1}{4} \sin \left(\frac{\alpha}{2}\right) \sin ^{3}\left(\frac{\theta_{0}}{2}\right) \sec ^{3}\left(\frac{\alpha+\theta_{0}}{2}\right)\left[3 \cos \left(\alpha+\theta_{0}\right)+\cos (\alpha)+3 \cos \left(\theta_{0}\right)+5\right] .
$$

We can now plot $a / R_{0}=\sin \theta_{0}$ as a function of $\delta$ for a fixed $\alpha$ as shown in Fig. 3. The medically-based limitation to small bulging angles, see Fig. 3, leads to a strong constraint between the relative volume increase and the size of the skull opening: even small volume changes on the order of a few percent require a large skull opening. This dependence may explain the significant amount of bone removed in clinical practice. The area of the removed bone flap can be readily obtained from the measurements of $a$ and $h_{0}$ as

$$
A=\pi\left(a^{2}+h_{0}^{2}\right)
$$

It is useful to provide quantitative estimates with realistic dimensions. We will use a standard skull size of $R_{0}=9 \mathrm{~cm}$ corresponding to a volume of about $3053 \mathrm{~cm}^{3}$, much greater than the typical brain volume of about $1200 \mathrm{~cm}^{3}$ showing the extra volume available for the brain in the skull (obviously, the brain and skull are not spherical and there is plenty of extra volume due to the ventricles and the subarachnoid space). In clinical practice, malignant middle cerebral artery infarctions are believed to require an additional volume of 80 to $160 \mathrm{~cm}^{3}$ for a sufficient brain pressure release. Clinical guidelines typically recommend an anteroposterior craniectomy diameter opening $d=2 a$ between 12 and $15 \mathrm{~cm}$, as shown in Fig. 4, although the exact rationale for these numbers is rather unclear as numerous factors affect the clinical outcome $[39,42,12]$.

Fig. 4 shows the added volume in $\mathrm{cm}^{3}$ for a skull of radius $R_{0}=9 \mathrm{~cm}$ as a function of the diameter opening $d$ for different values of the bulging angle $\alpha$. The graphs demonstrate the noticeable sensitivity of the craniectomy opening on the prescribed bulging angle and the range of relative volume increase in dependence of the craniectomy diameter. Even for 


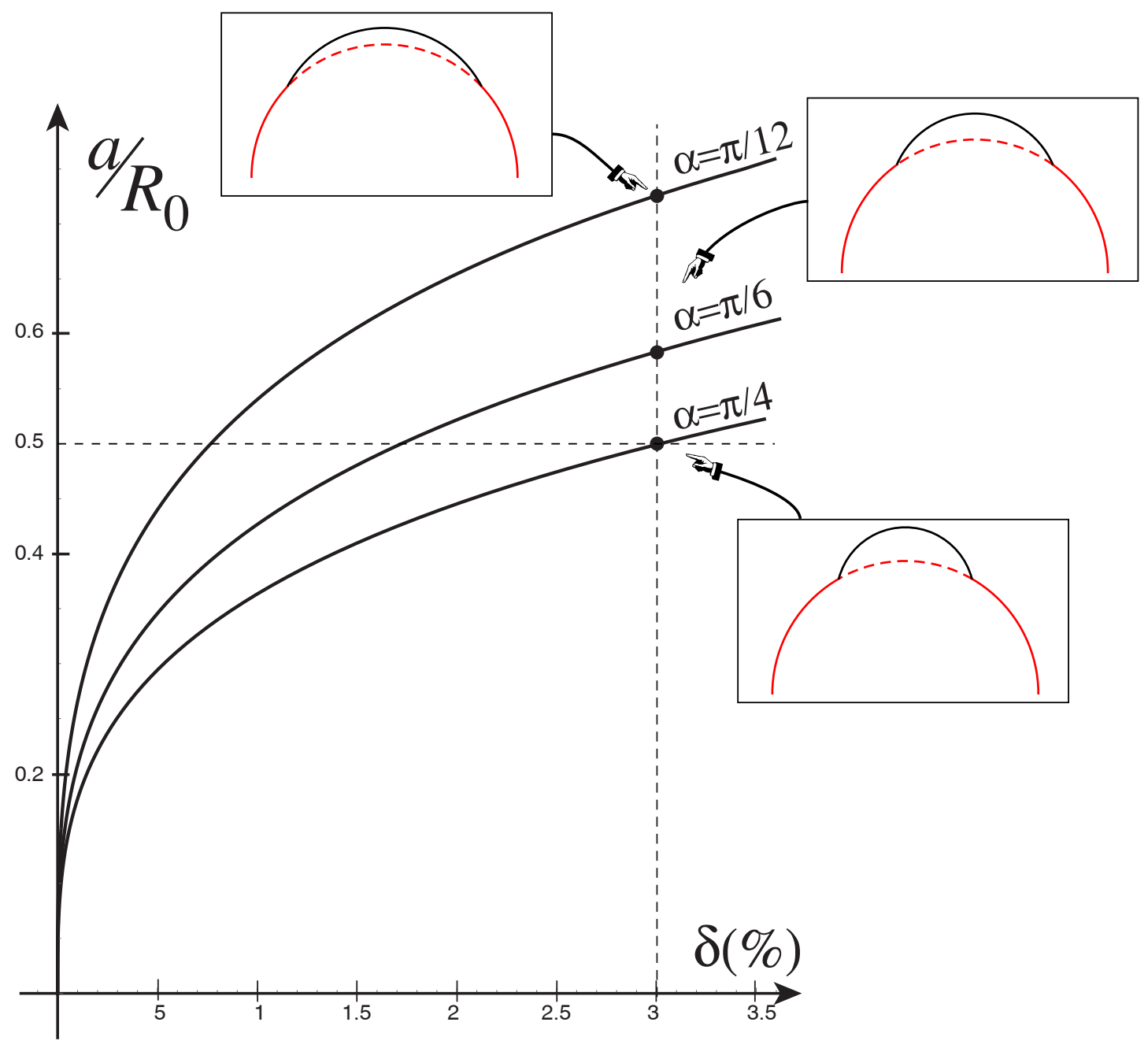

Figure 3: Opening size (measured as a fraction of the brain radius) as a function of the relative volume increase (in \%). For example a $3 \%$ volume increase with a fixed bulging angle of $\alpha=\pi / 4=45^{\circ}$ requires a relatively large opening of $x \approx R_{0} / 2$.

a small but physiological realistic variation of $d$ from 12 to $15 \mathrm{~cm}$ reported in the literature, our model predicts a large range for the added volume. Finally, we note that, within this simple description, the maximum stretch occurs along a line originally oriented along the vertical axis denoted by $h_{0}$ in Fig. 3 and stretched to $h_{1}$ after swelling. Then, the maximal stretch is given by

$$
\lambda_{\max }=\frac{R_{0}-h_{0}+h_{1}}{R_{0}}=\cos \left(\frac{\alpha-\theta_{0}}{2}\right) \sec \left(\frac{\alpha+\theta_{0}}{2}\right),
$$

and it is easy to appreciate from this last relationship that the maximum stretch increases rapidly with the bulging angle. 

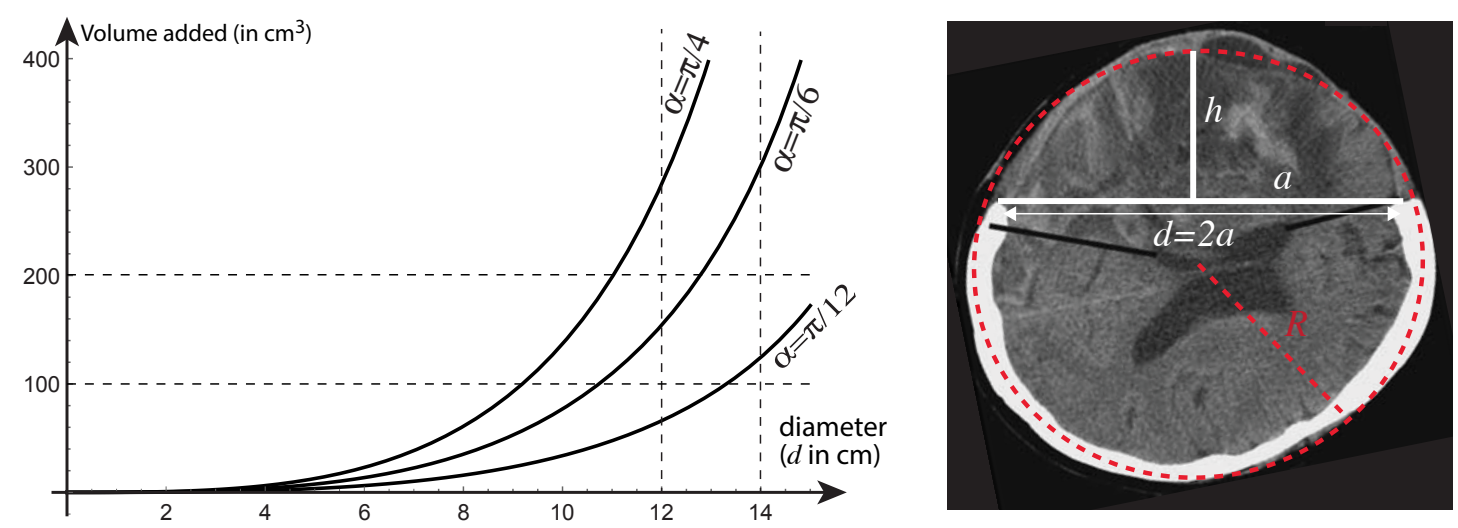

Figure 4: Relation between added volume, opening diameter $d$, and bulging angle $\alpha$. Right: Volume added for a skull of radius $R_{0}=9 \mathrm{~cm}$ for bulging angles $\alpha$ of $15^{\circ}, 30^{\circ}$, and $45^{\circ}$. Left: A computed tomography scan and the basic parameters used for geometric estimates [13].

\section{Mechanical models for half-space geometry}

The geometric estimates obtained in the previous section are based on a prescribed shape of the bulge and do not provide information on the stress and strain field that emerge during swelling. These mechanical quantities are particularly important to determine regions of potential tissue damage across length scales. The combined shear and compressive stress state at the contact point between the brain and the skull may generate herniation. On the axon level, fiber stretch may induce diffuse axonal damage. The evaluation of these stresses is a formidable task due to the complex geometry of the skull and the contact constraints between brain tissue and bone. Here, we start with an idealized geometry and consider the mechanical problem of bulging of a linear elastic half-space through a finite opening of width $2 a$, as shown in Fig. 5, similar to the combined experimental and numerical study of a similar geometry presented by Fletcher et al. [37]. In our study, we consider the twodimensional plane strain problem and the three-dimensional axisymmetric problem. These two geometric configurations allow for a complete mathematical description of stress and strain, that can be compared with the analytical three-dimensional axisymmetric solution and with finite-element simulations.

The bulging problem is reminiscent of the classic punch problem of indenting an infinite half-space with a finite object at a given force. Indeed, the bulging problem could be considered as a reverse punch in which the boundary conditions are inverted, that is, a force is applied by an infinite object with a central opening. Because the punch problem has important applications in indentation testing, there is an extensive literature on the subject with many variations $[43,44,45]$. Although it is likely that a solution may have been presented in the Russian literature [46], to the best of our knowledge, no explicit analytical treatment of the bulging problem exists. We also note that the solution to the bulging problem can be obtained by taking the proper limit of the annular punch problem [47, 48, 49, 50, 51] when the outer radius goes to infinity. However, there is no closed-form solution for this particular three-point boundary-value problem and it is more informative to completely solve a simple problem than to take the limit of a complicated one. We also note that bulging serves as a key indicator of the Poynting effect [52] in classic experiments on shear loading of rubber 
materials by Rivlin [53], and was also investigated numerically and experimentally in brain tissues [54].

It is not clear that a treatment of the bulging problem within the theory of linear elasticity is satisfactory, as we have noted in the previous discussion of possibly large local stresses and strains. However, we know that the solution of the indentation problem in linear elasticity is robust and provides excellent estimates. Our strategy here is to first obtain exact solutions and then compare them to finite-element solutions of the fully nonlinear problem to assess their validity. This direct comparison also provides a range of parameter values within which the linear solution serves as a satisfactory approximation of the nonlinear solution.

Our analytical solution will be obtained by considering the deformation of a half-space occupying the open domain $\Omega=\left\{\mathbf{x}=(x, y, z) \in \mathbb{R}^{3} \mid z>0\right\}$ with boundary $\Gamma=\{(x, y, z) \in$ $\left.\mathbb{R}^{3} \mid z=0\right\}$. The deformation is described by a displacement field $\mathbf{u}=\left(u_{x}, u_{y}, u_{z}\right): \Omega \rightarrow \mathbb{R}^{3}$ such that a point originally at $\mathbf{x} \in \Omega$ displaces to a point at $(\mathbf{x}+\mathbf{u}) \in \mathbb{R}^{3}$. From the displacement field $\mathbf{u}$, we define the (infinitesimal) strain tensor $\mathbf{E}$ as

$$
\mathbf{E}=\frac{1}{2}\left(\nabla \mathbf{u}+(\nabla \mathbf{u})^{\top}\right)
$$

The material is assumed to be a compressible, isotropic, initially unstressed, and linearly elastic with a Young's modulus $E$ and a Poisson's ratio $\nu$. Defining $\mathbf{T}$ as the Cauchy stress tensor, the constitutive relationship between stress and strain is given by

$$
\mathbf{T}=\frac{E}{1+\nu}\left(\mathbf{E}+\frac{\nu}{1-2 \nu}(\operatorname{tr} \mathbf{E}) \mathbf{1}\right)
$$

where $\mathbf{1}$ is the identity tensor.

The boundary is split into two subsets where the elastic material is either in contact $\left(\Gamma_{\mathrm{c}}\right)$ or traction free $\left(\Gamma_{\mathrm{f}}=\Gamma \backslash \Gamma_{\mathrm{c}}\right)$. Away from the opening of size $2 a$, the shape of the constrained region is given by a function $u_{\mathrm{c}}=u_{\mathrm{c}}(x, y)$ for $(x, y, 0) \in \Gamma_{r} \subseteq \Gamma$. The contact region $\Gamma_{\mathrm{c}}$ is then defined as the interior of the subset of $\Gamma_{r}$ such that $u_{z}(x, y)=u_{\mathrm{c}}(x, y)$. We further assume that the contact is frictionless so that there is no tangential traction.

For a given constraint $u_{\mathrm{c}}$, the bulging problem consists in finding the displacement $\mathbf{u}$ and the contact region $\Gamma_{\mathrm{c}}$, such that

$$
\begin{array}{ll}
\operatorname{div} \mathbf{T}=\mathbf{0}, & \mathbf{x} \in \Omega, \\
\mathbf{T n}=\mathbf{0}, & (x, y, 0) \in \Gamma_{\mathrm{f}}, \\
u_{z}(x, y)=u_{\mathrm{c}}(x, y), & (x, y, 0) \in \Gamma_{\mathrm{c}}, \\
\mathbf{T n}-(\mathbf{n} \cdot \mathbf{T n}) \mathbf{n}=\mathbf{0}, & (x, y, 0) \in \Gamma_{\mathrm{c}},
\end{array}
$$

where $\mathbf{n}$ denotes the outward unit normal to $\Omega$ on $\Gamma$ and the traction vector on $\Gamma$ is given by Tn. The last condition represents the frictionless constraint on the contact region.

\subsection{The two-dimensional bulging problem}

We consider an isotropic linearly elastic half-space under plane-strain conditions as shown in Fig. 5. Since the same setup is also valid for plane-stress conditions via rescaling the elastic parameters, it will not be studied explicitly here. For the plane-strain case, we assume that 


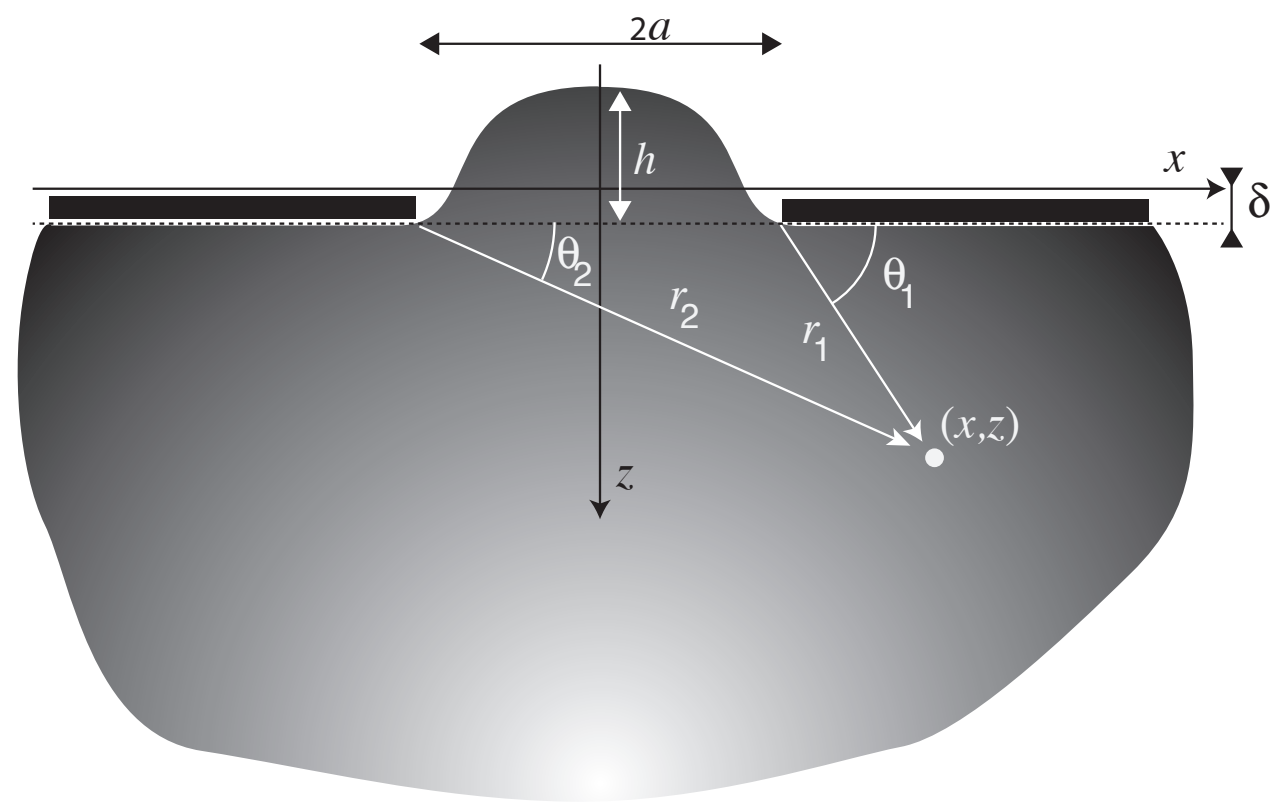

Figure 5: Two-dimensional bulging problem. The half-space is compressed by a plate, with an opening of radius $a$ in the middle, and displaced along the $z$-axis by magnitude $\delta$. Note that the position and coordinates of the point $(x, z)$ denote its location in the reference configuration.

the displacements only take place in the $x-z$ plane such that $u_{y}=0$. This condition implies that $E_{x y}=E_{y y}=E_{z y}=0$ for all $\mathbf{x} \in \Omega$.

A starting point for the contact analysis is the classic Cerruti-Flamant solution for a half-space under a single normal point load $P$ at the origin $[55,56,57]$. Then, for a point located at $x=r \cos \theta, z=r \sin \theta$, the radial stress, $T_{r r}=\mathbf{T e}_{r}$ with $\mathbf{e}_{r}=(\cos \theta, 0, \sin \theta)$, is the unit radial vector, is simply given by

$$
T_{r r}=-\frac{2 P}{\pi} \frac{\sin \theta}{r},
$$

whereas the other stress components in polar coordinates vanish identically. In Cartesian coordinates, this solution reads

$$
\begin{aligned}
& T_{x x}=-\frac{2 P}{\pi} \frac{x^{2} z}{\left(x^{2}+z^{2}\right)^{2}}, \\
& T_{z z}=-\frac{2 P}{\pi} \frac{z^{3}}{\left(x^{2}+z^{2}\right)^{2}}, \\
& T_{x z}=0 .
\end{aligned}
$$

We can use this point solution as a Green's function for the bulging problem and consider a distributed normal force $p(x) \mathrm{d} x$ on an element $\mathrm{d} x$ at each point on the surface. Therefore, in the absence of tangential loads acting on the surface, these elementary solutions can be 
readily integrated to obtain

$$
\begin{aligned}
& T_{x x}=-\frac{2 z}{\pi} \int_{-\infty}^{\infty} \frac{p(s)(x-s)^{2}}{\left((x-s)^{2}+z^{2}\right)^{2}} \mathrm{~d} s \\
& T_{z z}=-\frac{2 z^{3}}{\pi} \int_{-\infty}^{\infty} \frac{p(s)}{\left((x-s)^{2}+z^{2}\right)^{2}} \mathrm{~d} s \\
& T_{x z}=-\frac{2 z^{2}}{\pi} \int_{-\infty}^{\infty} \frac{p(s)(x-s)}{\left((x-s)^{2}+z^{2}\right)^{2}} \mathrm{~d} s .
\end{aligned}
$$

Defining the displacement at the surface to be $\overline{\mathbf{u}}=\left(\bar{u}_{x}, \bar{u}_{z}\right)$, these relations can be used together with (12-13) to obtain a simple relationship between the applied load and the surface displacement gradient,

$$
\begin{aligned}
& \frac{\partial \bar{u}_{x}}{\partial x}=-\frac{(1-2 \nu)(1+\nu)}{E} p(x) \\
& \frac{\partial \bar{u}_{z}}{\partial x}=-\frac{2\left(1-\nu^{2}\right)}{\pi E} \int_{-\infty}^{\infty} \frac{p(s)}{x-s} \mathrm{~d} s .
\end{aligned}
$$

In our case, we consider a uniform displacement $\delta$, except in a region centered at the origin where $p(x)=0$, that is

$$
\frac{\partial \bar{u}_{z}}{\partial x}=0, \quad|x|>a
$$

Inserting this condition in (26), the normal load that guarantees this displacement must satisfy

$$
\int_{-\infty}^{-a} \frac{p(s)}{x-s} \mathrm{~d} s+\int_{a}^{\infty} \frac{p(s)}{x-s} \mathrm{~d} s=0 .
$$

This equation for $p(x)$ is a singular integral equation of the first kind. Its homogeneous solution is of the form

$$
p(x)= \begin{cases}\frac{c_{-}}{\sqrt{-x-a}} & x \leq-a, \\ \frac{c_{+}}{\sqrt{x-a}} & x \geq a .\end{cases}
$$

From symmetry considerations, we must have $c_{-}=c_{+}=c \sqrt{a} E / 4$, where $c$ is an arbitrary dimensionless constant, and the normal load distribution may be re-written in the following form

$$
p(x)= \begin{cases}0 & |x|<a, \\ \frac{c E}{4} \sqrt{|x|-a} & |x| \geq a .\end{cases}
$$

Once the load is known, we can directly obtain the shape of the bulge in the gap by integrating Equation (26) once. Explicitly, it reads

$$
\bar{u}_{z}=\delta-c \sqrt{a}\left(\nu^{2}-1\right)[\sqrt{2 a}-\sqrt{a-x}-\sqrt{a+x}], \quad|x|<a .
$$

Note that the value of $\delta$ does not enter in the computation of the shape and can be set to zero without loss of generality. The area $A_{0}$ of a bulge is then

$$
A_{0}=\frac{2}{3} \sqrt{2} a^{2} c\left(1-\nu^{2}\right)
$$




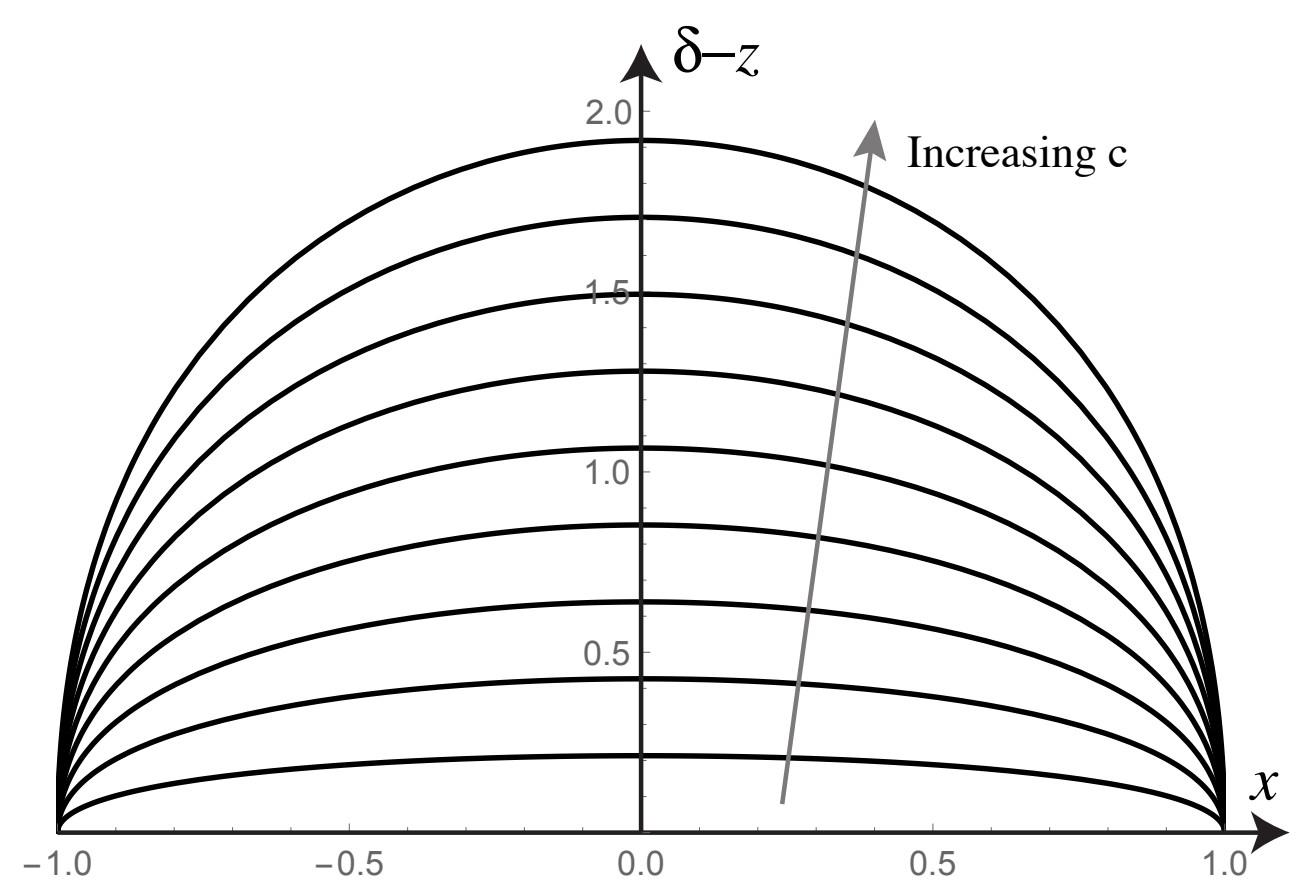

Figure 6: A series of two-dimensional plane-strain bulges obtained for increasing values of $c$ from 0.4 to 3.6 in increments of 0.4 , where $a=1$ and $\nu=0.3$.

The profile of the bulge is shown in Fig. 6 for increasing values of $c$.

Since we have an explicit expression for the normal load distribution on the surface, we can compute the stresses at all points within the material by integrating Equations (22-24). A compact form of these integrals is obtained by using the double polar representation shown in Fig. 5 whenever convenient (that is a point is represented as $x=a+r_{1} \cos \theta_{1}, y=r_{1} \sin \theta_{1}$ for all integrals on the positive axis and $x=-a+r_{2} \cos \theta_{2}, y=r_{2} \sin \theta_{2}$ for integrals on the negative part of the axis). After simplification, the stresses can be written as

$$
\begin{aligned}
& T_{x x}=-\frac{c \sqrt{a} E}{16}\left(\frac{3 \cos \left(\frac{\theta_{1}}{2}\right)+\cos \left(\frac{5 \theta_{1}}{2}\right)}{\sqrt{r_{1}}}+\frac{3 \sin \left(\frac{\theta_{2}}{2}\right)+\sin \left(\frac{5 \theta_{2}}{2}\right)}{\sqrt{r_{2}}}\right), \\
& T_{z z}=\frac{c \sqrt{a} E}{4}\left(\frac{\cos ^{3}\left(\frac{\theta_{1}}{2}\right)\left(2 \cos \left(\theta_{1}\right)-3\right)}{\sqrt{r_{1}}}-\frac{\sin ^{3}\left(\frac{\theta_{2}}{2}\right)\left(2 \cos \left(\theta_{2}\right)+3\right)}{\sqrt{r_{2}}}\right), \\
& T_{x z}=-\frac{c \sqrt{a} E}{16}\left(\frac{\sin \left(\frac{\theta_{1}}{2}\right)-\sin \left(\frac{5 \theta_{1}}{2}\right)}{\sqrt{r_{1}}}+\frac{\cos \left(\frac{5 \theta_{2}}{2}\right)-\cos \left(\frac{\theta_{2}}{2}\right)}{\sqrt{r_{2}}}\right) .
\end{aligned}
$$

It should be noted, that our formulation recovers the well-known scaling law $1 / \sqrt{r}$ of the stress around the singularity point for the classic punch problem [45].

We are particularly interested in identifying regions in the proximity of the contact point where large stresses are likely to induce tissue damage. Since stresses are described by a tensor, we could explore different alternative stress measures as functions of the three stresses $T_{x x}, T_{z z}$, and $T_{x z}$. Based on medical evidence that links diffuse axonal damage to increased shear loading of brain tissue, we associate damage with shear stress, while acknowledging that other stress measures, such as the von Mises stress or the maximum 
principal stress could be used alternatively. In Fig. 7, we show the shear stress with respect to the reference configuration. While we chose particular values for this figure, this plot is generic as the color bar and the scale length can be rescaled for other systems. In particular, we recognize characteristic drop shapes for the level sets of shear stress emerging from the points of contact where the stresses diverge at the edge of the plate. At each of these contact points, there exist two drop-like regions of high stresses (in absolute value) where we can expect a high risk of damage. We call these regions damage drops and study them in further detail.

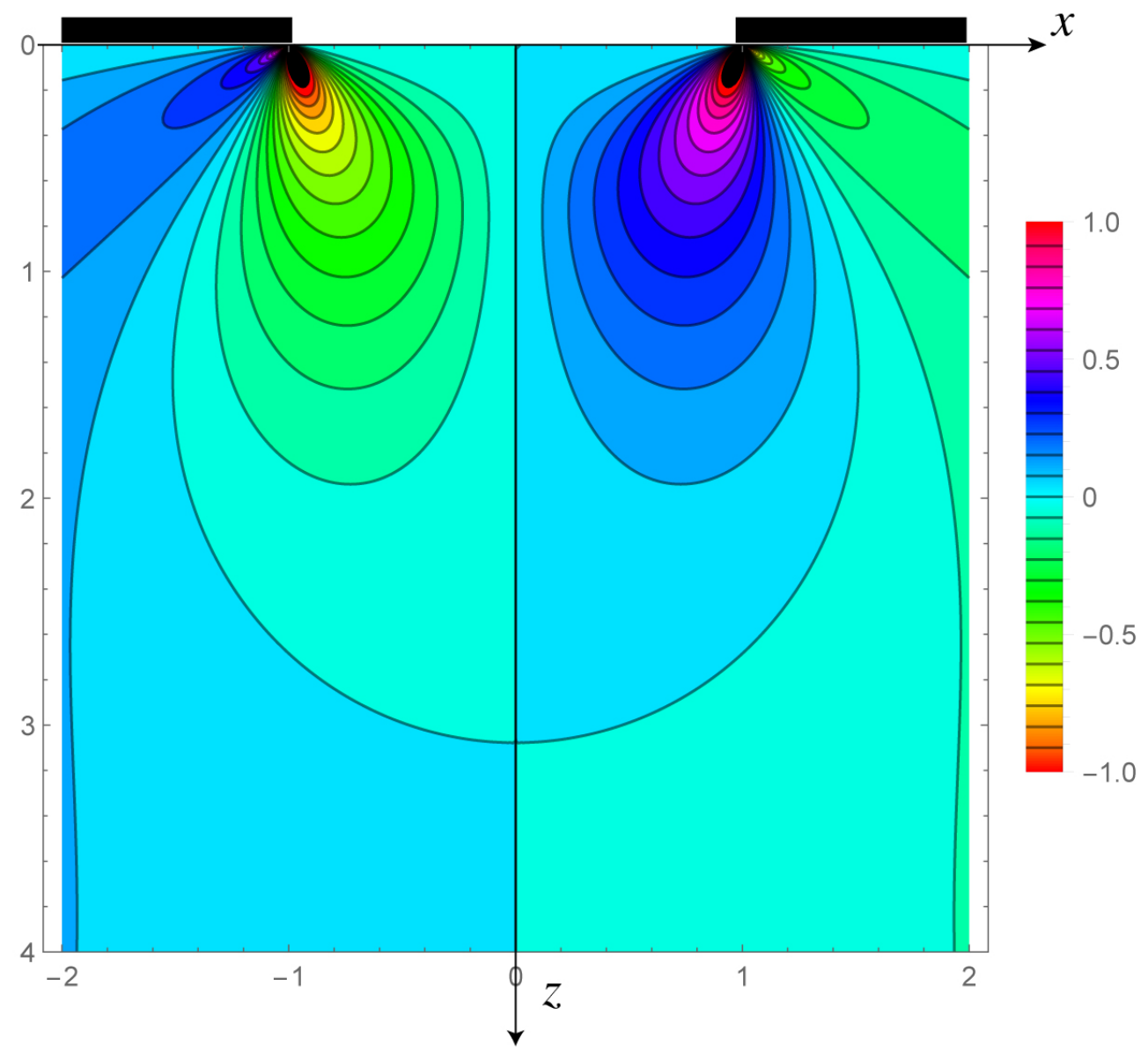

Figure 7: Contour plot of the shear stress in the reference configuration for the plane strain bulging problem.

Let $\tau_{\text {crit }}>0$ be the critical value of shear stress past which damage is expected. That is, damage occurs for

$$
\left|T_{x z}\right|>\tau_{\text {crit }} .
$$

For small enough values of the amplitude $c$, an approximation of the damage drops can be obtained by neglecting the effect of the other edge. That is, we restrict our attention to the stresses created by the plate on the positive $x$-axis. In this case, the expression for $T_{x z}$ only depends on $\left(r_{1}, \theta_{1}\right)$ so that a suitable approximation is given by

$$
T_{x z}^{\mathrm{a} p p}=-\frac{c E}{16}\left(\frac{\sin \left(\frac{\theta_{1}}{2}\right)-\sin \left(\frac{5 \theta_{1}}{2}\right)}{\sqrt{r_{1}}}\right) .
$$


We introduce a dimensionless parameter, $K$, to measure the extent of the damage zone with respect to the ratio of Young's modulus to the critical shear stress:

$$
K=\frac{c E}{16 \tau_{\text {crit }}} .
$$

Then the damage drops, shown in Fig. 8, are defined by the polar curve

$$
r_{1}\left(\theta_{1}\right)=K^{2}\left(\sin \frac{\theta_{1}}{2}-\sin \frac{5 \theta_{1}}{2}\right)^{2} \quad 0 \leq \theta_{1} \leq \pi .
$$

The orientations of the two damage drops with respect to the horizontal axis are given by the angles

$$
\begin{aligned}
& \alpha_{+}=2 \tan ^{-1}\left(\frac{1}{2} \sqrt{\frac{1}{3}(13+\sqrt{145})}\right) \approx 111^{\circ}, \\
& \alpha_{-}=2 \tan ^{-1}\left(\frac{1}{2} \sqrt{\frac{1}{3}(13-\sqrt{145})}\right) \approx 32^{\circ} .
\end{aligned}
$$

The damage drops provide a suitable approximation for $\tau_{\text {crit }} / E$ large enough (or equivalently $c$ small enough) as shown in Fig. 8. Their areas provide an upper bound for the damage zone in that regime. These areas are given explicitly by

$$
\begin{aligned}
& A_{+}=\frac{3(243 \sqrt{3}+280 \pi) K^{4}}{1120} \approx 3.48357 K^{4}, \\
& A_{-}=-\frac{3(243 \sqrt{3}-140 \pi) K^{4}}{1120} \approx 0.0507177 K^{4} .
\end{aligned}
$$

Of particular interest is the relative growth of the total area of the four drops with respect to the area of the bulge itself, that is

$$
2 \frac{A_{+}+A_{-}}{A_{0}}=\frac{3^{3} \pi}{2^{19} \sqrt{2}} \frac{E^{4} c^{3}}{\tau_{\text {crit }}^{4}\left(1-\nu^{2}\right)} \approx 0.0000572004 \frac{E^{4} c^{3}}{\tau_{\text {crit }}^{4}\left(1-\nu^{2}\right)} .
$$

The important scaling here is that the area of damage increases as the fourth power of the solution amplitude, $c$, whereas the bulge area only increases linearly. Therefore, we expect the damage drops to grow very quickly once they reach a non-negligible size. For instance, a bulge with the largest value of $c=3.6$ and $\nu=0.3$ in Fig. 6 with $\tau_{\text {crit }}=E / 2$ would create a large damage area of about $9 \%$ of the bulge area.

\subsection{The three-dimensional axisymmetric bulging problem}

Next, we turn to the analysis of the bulging problem under axisymmetric conditions in cylindrical coordinates. We consider the deformation of an elastic half-space when constrained everywhere except at a circular opening of radius $a$ centered at the origin on the boundary of the half-space. We assume that the half-space is isotropic and that the contact is frictionless. We exploit the equivalence between the problem of finding a bulge due to uniform 

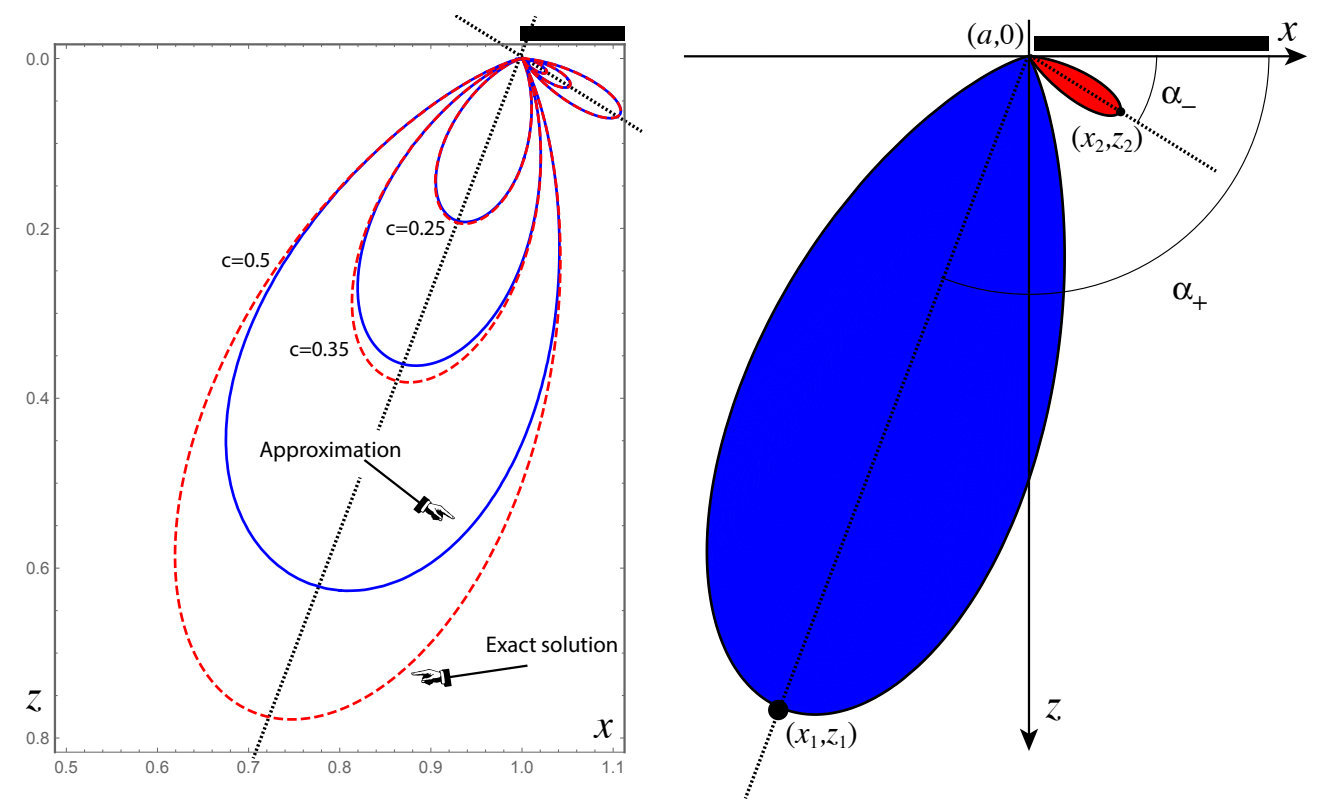

Figure 8: Approximation of the size and inclination of the damage drops valid for values of $K$ sufficiently small, obtained by neglecting the effect of the other opening. Left. Comparison of the approximation (solid blue line) with the exact solution (dashed red line) for $a=c=$ $\tau_{\text {crit }}=1, E=16$ and $c=0.25,0.35,0.5$. Right. Approximated damage drop shapes valid for small $c$.

swelling on a fixed boundary and the problem of determining the shape obtained by displacing the contact zone at the boundary of the half-space vertically downward. A finite-element simulation of the problem is shown in Fig. 9.

Compared to the plane-strain problem, a different set of analytical methods is used to solve the axisymmetric problem. The first step is to transform the original formulation of the problem in terms of a potential by using the Papkovich-Neuber formulation. In the usual cylindrical coordinates $(r, \theta, z)$, we introduce a potential $\phi(r, z)$ such that

$$
\mathbf{u}=\frac{1+\nu}{E}\left[4(\nu-1) \boldsymbol{\omega}+\nabla\left(\mathbf{x} \cdot \boldsymbol{\omega}+\frac{\phi}{1-2 \nu}\right)\right], \quad \text { where } \boldsymbol{\omega}=\mathbf{e}_{z} \frac{\partial \phi}{\partial z}
$$

and $\mathbf{e}_{z}$ is the unit vector along the $z$-axis. If we choose the potential to be a harmonic function, that is

$$
\triangle \phi \equiv \frac{\partial^{2} \phi}{\partial z^{2}}+\frac{1}{r} \frac{\partial}{\partial r} r \frac{\partial \phi}{\partial r}=0
$$

then the Cauchy equation, $\operatorname{div} \mathbf{T}=\mathbf{0}$, is automatically satisfied. Since the displacements are given in terms of the potential $\phi$, all derived quantities such as stress, strain, and position can be obtained from $\phi$. The key is then to choose among the large class of harmonic functions, the one particular function that satisfies the boundary conditions

$$
\begin{array}{ll}
T_{r z}=T_{\theta z}=0, & z=0, \\
T_{z z}=0, & 0 \leq r<a, z=0, \\
\bar{u}_{z}=\delta(r), & r \geq a, z=0 .
\end{array}
$$



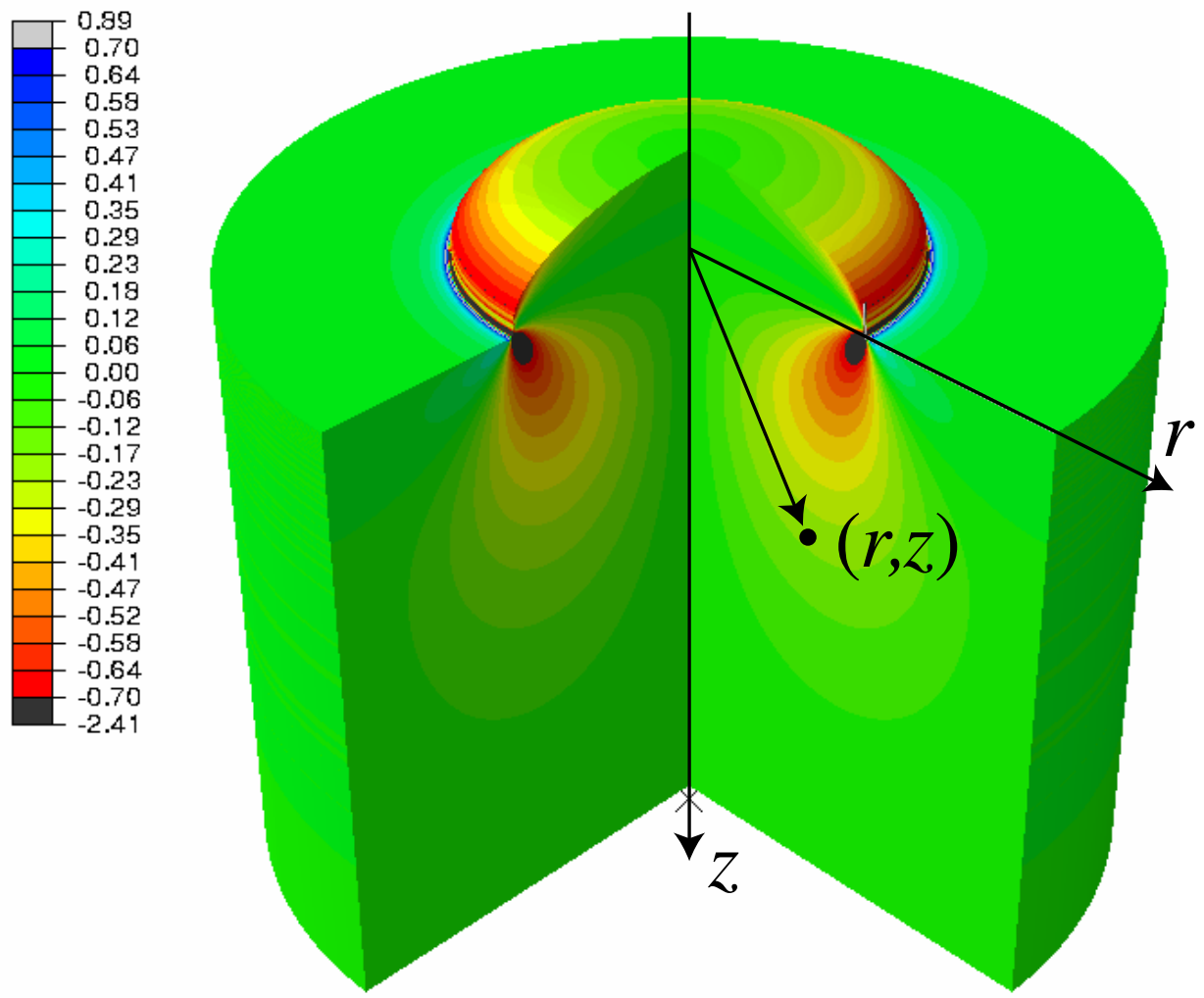

Figure 9: Bulging simulation in a cylindrical geometry with a cylindrical coordinate system.

On the surface $z=0$, the conditions $T_{r z}=T_{\theta z}=0$ are automatically satisfied and the vertical displacements and tractions are given in terms of $\phi$ by

$$
T_{z z}=-\frac{\partial^{2} \phi}{\partial z^{2}}, \quad \bar{u}_{z}=2 \frac{\nu^{2}-1}{E} \frac{\partial \phi}{\partial z}+C_{z}
$$

where $C_{z}$ is an arbitrary constant corresponding to an arbitrary rigid-body vertical translation of the contact plate. Therefore, our problem is to find a harmonic function $\phi=\phi(r, z)$ such that

$$
\begin{array}{ll}
\frac{\partial^{2} \phi}{\partial z^{2}}=0, & 0 \leq r<a, z=0, \\
\frac{\partial \phi}{\partial z}=\frac{E}{2\left(\nu^{2}-1\right)} \delta(r), & r \geq a, z=0 .
\end{array}
$$

An elegant way to solve this problem $[58,59,60]$ consists in introducing an unknown function $g(s)$ and an integral representation for the potential $\phi$ of the form

$$
\phi=\Im \mathrm{m}\left(\int_{a}^{\infty} g(s) \mathcal{F}(r, z, s) \mathrm{d} s\right)
$$

where

$$
\mathcal{F}(r, z, s)=\log \left(\sqrt{r^{2}+(z+i t)^{2}}+s+i t\right) .
$$


The condition (51) is then automatically satisfied and condition (52) is now a single integral equation

$$
-\int_{r}^{\infty} \frac{g(s)}{\sqrt{s^{2}-r^{2}}} \mathrm{~d} s=\frac{E}{2\left(\nu^{2}-1\right)} \delta(r), \quad r \geq a .
$$

On the left-hand-side of this equation, we recognize the Abel transform of the function $g(s) / s$. An inverse Abel transform of this relation gives the function $g(s)$ in terms of $\delta(r)$,

$$
g(s)=\frac{E}{\pi\left(\nu^{2}-1\right)} \frac{\mathrm{d}}{\mathrm{d} s} \int_{s}^{\infty} \frac{r \delta(r)}{\sqrt{r^{2}-s^{2}}} \mathrm{~d} r, \quad r \geq a .
$$

\subsubsection{Bulging with a constant contact profile}

In general, the point $a$ at which the elastic solid and the plate separate upon increasing deformation is dependent on the respective contact profile $\delta(r)$. To simplify the determination of this specific point, we consider the simplifying assumption $\delta(r)=\delta$ for $r>a$. This constant profile guarantees that $a$ is fixed by the geometry of the contact plate and does not change with the deformation. However, for a constant profile, the usual Abel transform (56) does not converge. This problem can be circumvented, first by defining a one-parameter family of functions

$$
g_{b}(s)= \begin{cases}\frac{E}{2\left(1-\nu^{2}\right)} \frac{h}{a} s, & 0<s<b \\ 0 & s \geq b\end{cases}
$$

and accordingly,

$$
\phi_{b}=\frac{E h}{\pi a\left(\nu^{2}-1\right)} \Im \mathrm{m}\left(\int_{a}^{b} s \mathcal{F}(r, z, s) \mathrm{d} s\right) .
$$

Second, by choosing $C_{z}=(\delta-h b / a)$ in (50) and taking the limit $b \rightarrow \infty$, we verify that the boundary conditions on the vertical displacements in the contact region are satisfied since

$$
\bar{u}_{z}=\delta+\lim _{b \rightarrow \infty}\left(-\int_{r}^{b} \frac{h s}{\sqrt{s^{2}-r^{2}}} \mathrm{~d} s-\frac{h}{a} b\right)=\delta, \quad r>a .
$$

The displacements in the bulge region are then given by

$$
\bar{u}_{z}=\delta+\lim _{b \rightarrow \infty}\left(-\int_{a}^{b} \frac{h s}{\sqrt{s^{2}-r^{2}}} \mathrm{~d} s-\frac{h}{a} b\right)=\delta-\frac{h}{a} \sqrt{a^{2}-r^{2}}, \quad r<a .
$$

Without loss of generality, we choose $\delta=0$ in the above expression to obtain the bulge shape

$$
\bar{u}_{z}(r)=-\frac{h}{a} \sqrt{a^{2}-r^{2}}, \quad r<a .
$$

Geometrically, the bulge is a hemisphere, vertically rescaled by a factor $h / a$. The displacements at all points in the body can be computed from the general relationship between the displacements and the potential function,

$$
\begin{aligned}
& u_{z}=\frac{(1+\nu)}{E}\left(z \frac{\partial^{2} \phi}{\partial z^{2}}-2(1-\nu) \frac{\partial \phi}{\partial z}\right)+C_{z}, \\
& u_{r}=\frac{(1+\nu)}{E}\left(z \frac{\partial^{2} \phi}{\partial r \partial z}-(1-2 \nu) \frac{\partial \phi}{\partial r}\right)+C_{r} .
\end{aligned}
$$




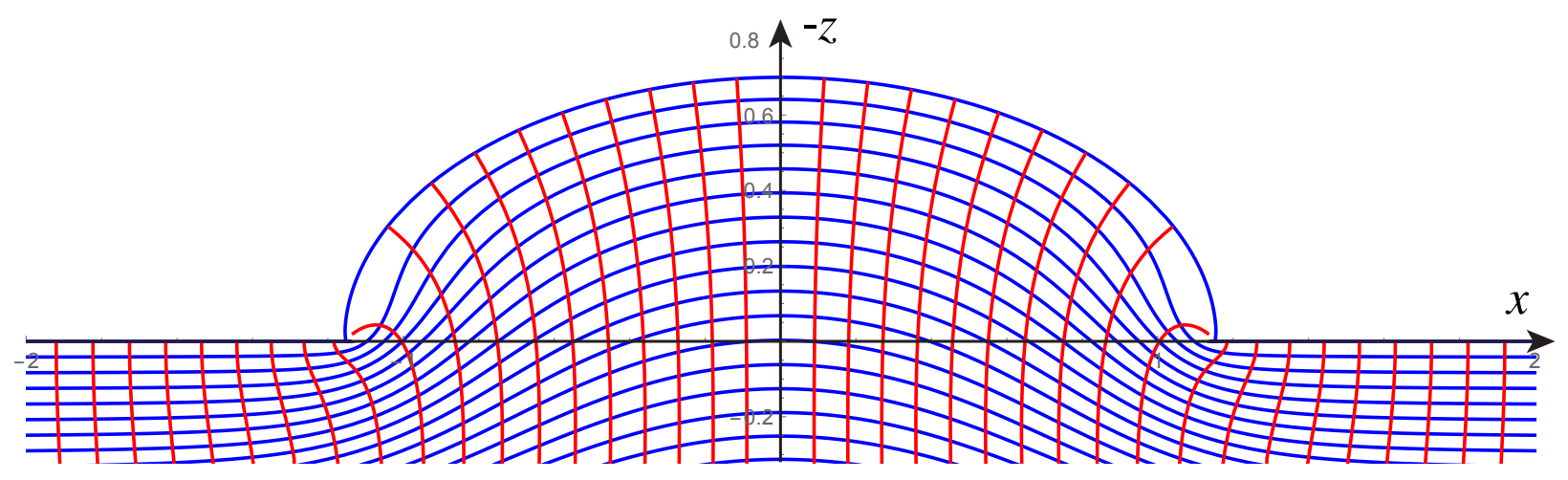

Figure 10: Displacements and bulge shape in the deformed configuration. The slice is taken at $y=0$ of the deformed half-space. The red initially vertical and blue initially horizontal curves correspond to the deformed lines of constant $r$ and constant $z$, respectively. Here, $a=1, \nu=0.3$, and $h=0.733$.

These relationships are understood in the same limit sense as the surface displacements, obtained by evaluating all quantities on the right-hand-sides up to a fixed value $b$, and taking the limit $b \rightarrow \infty$ with the proper choice of the constants to remove the divergence. These displacements are illustrated in Fig. 10 for the particular choice of $\nu=0.3$ and $h=0.733$.

To understand the range of validity of the linear approximation, we compare the surface profiles with finite-element simulations of an incompressible neo-Hookean material under the same contact conditions (note that the surface displacements do not depend on the choice of the Poisson ratio). The comparison shown in Fig. 11 suggests that the linear solution is an excellent approximation of the nonlinear solution for values of $h \lesssim a / 2$, which we take as the domain of validity. We also note that in this range, the two-dimensional solution (31) obtained under plane strain conditions, is also an excellent approximation of the profile as the two profiles are indistinguishable for $h / a$ small enough. For larger values of $h / a$, the linear solution looses its validity. There are two effects at play: First, large rotations are observed close to the contact point, see Fig. 10. Typically, the linear elastic solution fails to properly account for finite rotations. Second, the vertical boundary conditions at the point of contact necessary for the finite-element simulation play an increasingly important role. These conditions can also be implemented within the linear theory of elasticity by appropriately choosing $\delta(r)$ in (56). However, in this regime, the solution looses its generic features and the shape will depend strongly on the boundary conditions.

Since, the bulge is a solid of revolution, its volume can be directly obtained by rescaling the volume of a hemisphere,

$$
V_{\text {bulge }}=\frac{2}{3} \pi a^{2} h .
$$

If the tissue is nearly incompressible, this extra volume corresponds to the addition of mass due to fluid intake.

Next, we turn our attention to the stresses generated in bulging solids. Two components of the stress field are particularly relevant in the case of decompressive craniectomy: the shear stresses $T_{r z}$ and the vertical stresses $T_{z z}$. These components of the stress tensor are 


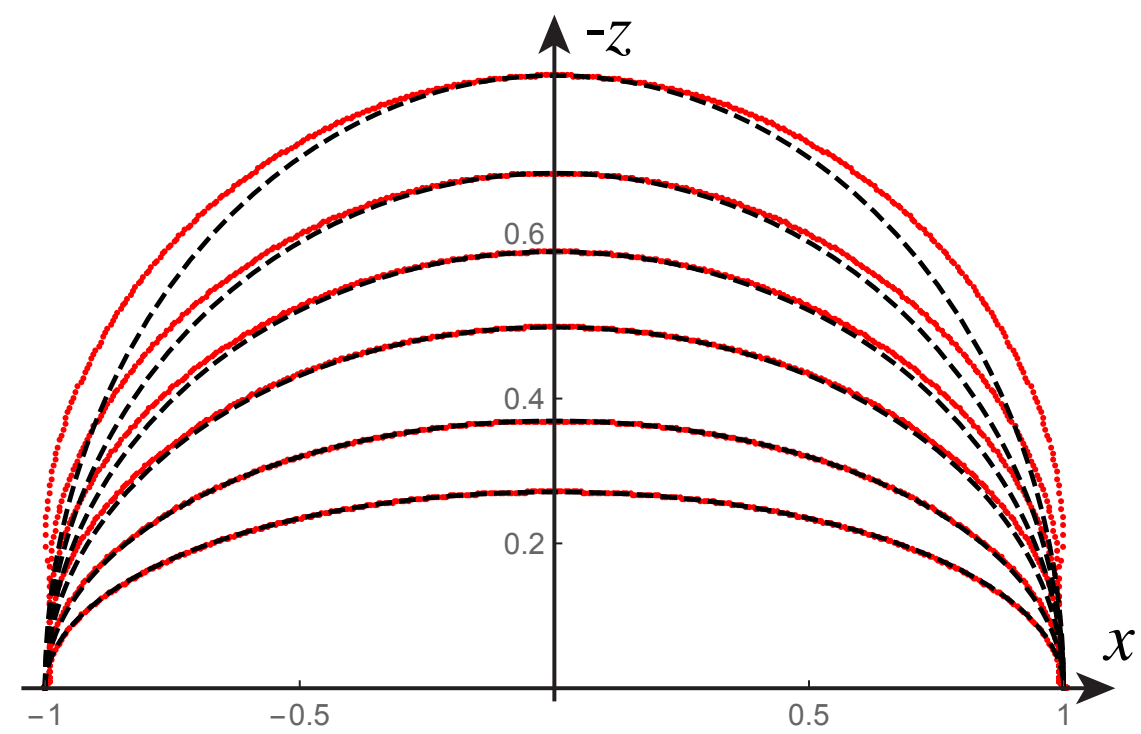

Figure 11: Comparison between nonlinear finite-element solution (red-dotted) and linear solution (black-dashed) for increasing values of $h \in\{0.27,0.37,0.50,0.60,0.71,0.85\}$ at $a=1$.

obtained from the potential as

$$
T_{r z}=z \frac{\partial^{3} \phi}{\partial \theta \partial z^{2}}, \quad T_{z z}=z \frac{\partial^{3} \phi}{\partial z^{3}}-\frac{\partial^{2} \phi}{\partial z^{2}} .
$$

First, we consider the shear stresses which are classically associated with the notion of damage in ductile materials. These stresses, shown in Fig. 12, also exhibit the characteristic damage drops found in the plane strain case. In analogy to the damage criterion (36), we now define the damage drops as regions of high absolute shear stress, such that

$$
\left|T_{r z}\right|>\frac{E}{1+\nu} \gamma_{\text {crit }}
$$

for a fixed maximal shear strain $\gamma_{\text {crit }}$. Unlike in the two-dimensional case, there is no simple characterization of the shear stresses. Nevertheless, we can study their asymptotic behavior close to the contact point. We expand the shear stresses (65) for small values of $\rho$, where $a \rho$ is the distance between a material point and the boundary point, such that $r=a(1+\rho \cos \alpha)$ and $z=a \rho \sin \alpha$. We consider the first two non-vanishing terms of this expansion obtained to order $\mathcal{O}\left(\rho^{3 / 2}\right)$ :

$$
T_{r z}^{\mathrm{app}}=\frac{E}{4 \sqrt{2}\left(1-\nu^{2}\right)} \frac{h}{a} \frac{1}{\sqrt{\rho}} \sin \alpha\left[\cos \frac{3 \alpha}{2}-\frac{\rho}{4}\left(6 \cos \frac{\alpha}{2}+\cos \frac{5 \alpha}{2}\right)\right]
$$

For large enough $\gamma_{\text {crit }}$, there are two damage drops defined by (66) as shown in Fig. 13. Remarkably, from the dominant terms in (67), the orientations of these two drops are given by the same expressions as in the two-dimensional case:

$$
\alpha_{ \pm}=2 \tan ^{-1}\left(\frac{1}{2} \sqrt{\frac{1}{3}(13 \pm \sqrt{145})}\right)
$$




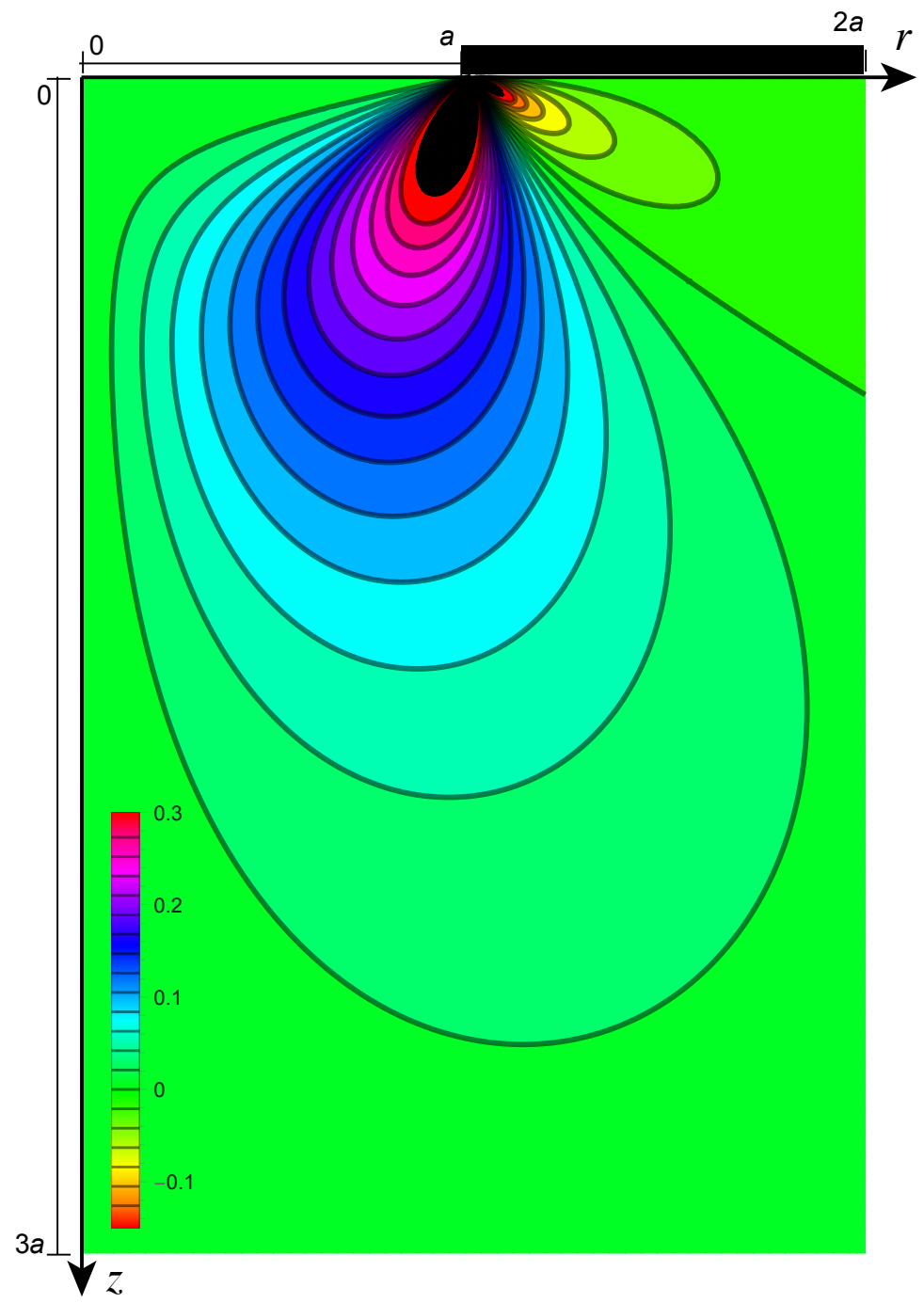

Figure 12: Contour map of shear stress in the reference configuration for $\nu=0.45, E=1$, and $h / a=1 / 2)$.

The total volume of these drops in this range is well approximated by

$$
V_{\text {drop }} \approx a^{3}\left(\frac{h^{2}}{a^{2}} \frac{0.01826}{\gamma_{\text {crit }}^{2}(1-\nu)^{2}}+\frac{h^{4}}{a^{4}} \frac{0.00033}{\gamma_{\text {crit }}^{4}(1-\nu)^{4}}\right) .
$$

We observe that in the range of validity, $h /\left(a \gamma_{\text {crit }}\right) \lesssim 0.5$, the volume of the damage drops increases initially with the height of the bulge as $(h / a)^{2}$.

So far, we have focused on tissue damage at the point of contact where the highest absolute shear stress is observed. Another important source of damage in brain trauma is associated with excessive stretch of axon fibers. Since we have neglected material anisotropy thus far, we have not included the mechanical effect of axon fiber direction in our model. However, if we assume that axon bundles are oriented normal to the surface in the reference configuration, we can investigate their length change by considering the stretch of vertical material lines. In terms of the harmonic potential, the vertical stretch $\lambda_{z}=1+\partial_{z} u_{z}$ is given 


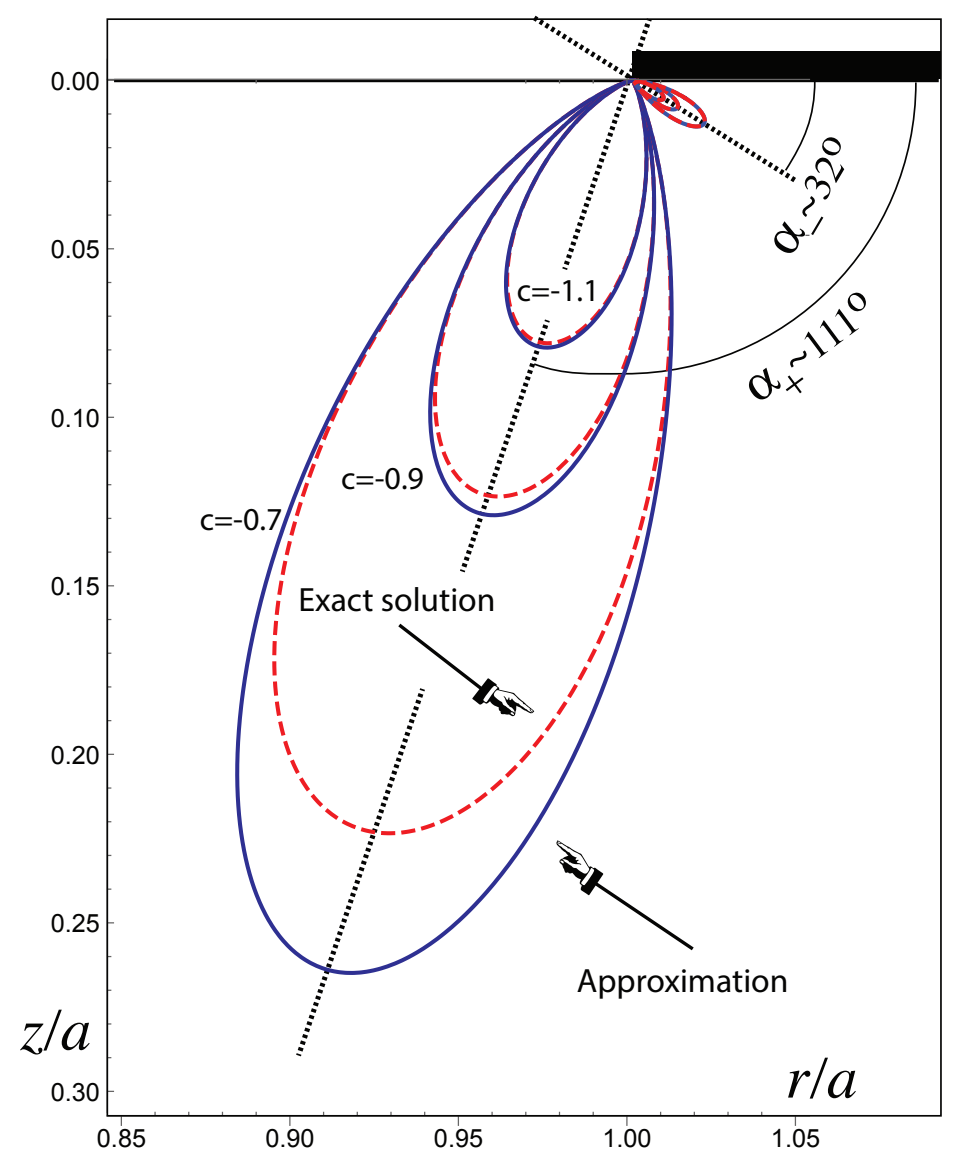

Figure 13: Approximation of the size and inclination of the damage drops. Comparison of the approximation (solid blue line) with the exact solution (red dashed line) for $c=\gamma_{\text {crit }} / h$, $\nu=0.45$, and $E h / a=1$.

by

$$
\lambda_{z}(z, \nu)=1+\frac{1+\nu}{E} \lim _{b \rightarrow \infty}\left(z \frac{\partial^{3} \phi_{b}}{\partial z^{3}}+(2 \nu-1) \frac{\partial^{2} \phi_{b}}{\partial z^{2}}\right) .
$$

As expected, the stretch is minimal at the point of contact with the same singularity in $1 / \sqrt{\rho}$ as the shear stress. The stretch is maximal on the axis of symmetry at $r=0$ as illustrated in Fig. 14. This maximum is located at $z_{\max }=a \sqrt{\nu /(2-\nu)}$. At this point, the stretch becomes maximal when the last term in (70) vanishes, that is, in the incompressible limit as $\nu \rightarrow 1 / 2$. In this limit, the stretch on the axis is simply given by

$$
\lambda_{z}(z, 1 / 2)=1+\frac{2 h z a^{2}}{\left(a^{2}+z^{2}\right)^{2}}
$$

with the maximal value

$$
\lambda_{\max }=\lambda_{z}\left(z_{\max }, 1 / 2\right)=\lambda_{z}\left(\frac{a}{\sqrt{3}}, 1 / 2\right)=1+\frac{3 \sqrt{3} h}{8 a} .
$$

This is an interesting generic result. As discussed in Section 1.3, axonal damage may appear for strains as low as 3-5\% and significant levels of cell injury occur when the axonal strains 
reach $20 \%$. From (72), we see that the stretch level of $\lambda_{\max }=1.2$ is achieved when $h / a \approx 0.3$. We conclude that significant levels of axonal damage will occur if $a$ is not large enough. As a simple estimate, if we limit the height of the bulge to a maximum of $h / a=0.3$, then from (64) the minimal size for $a$ is found to be

$$
a_{\min }=\left(\frac{5}{\pi} V_{\text {bulge }}\right)^{1 / 3} .
$$

This suggests that swelling volumes between 80 and $160 \mathrm{~cm}^{3}$ require a minimal diameter $2 a_{\min }$ of 10 to $12.6 \mathrm{~cm}$, respectively, which compares preferably with the opening diameters of about 12 to $15 \mathrm{~cm}$ recommended for surgical practice.

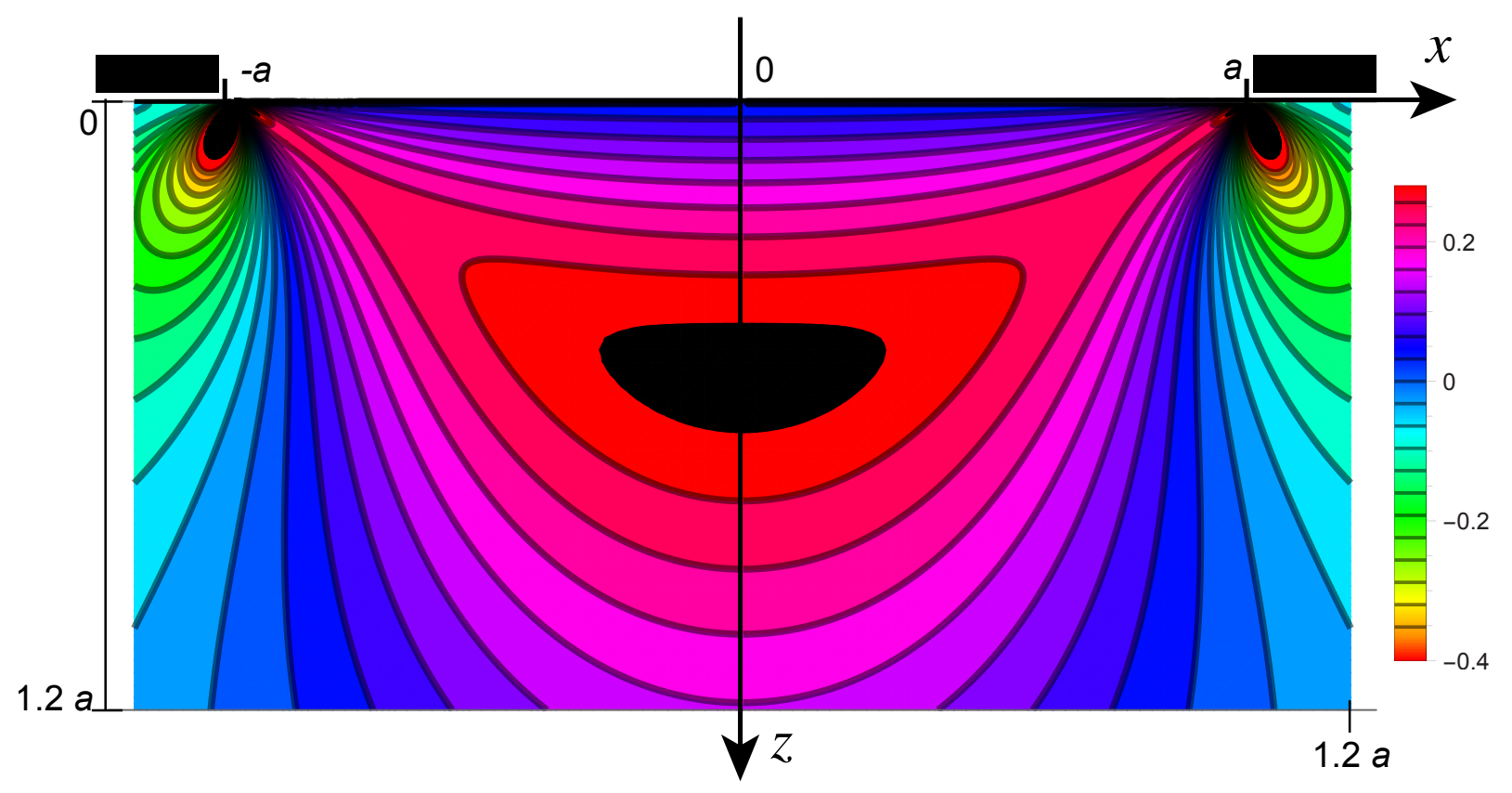

Figure 14: Vertical strains in the reference configuration. Strains take maximum values larger than $20 \%$ along the axis of symmetry at $x=0$. Here, $\nu=0.45, h=1 / 2$, and $E=1$.

\section{Conclusion}

Decompressive craniectomy is a highly invasive and complex surgical intervention that aims at an immediate release of elevated intracranial pressure to minimize potential brain damage due to ischemia or excessive herniation. The removal of the skull allows the brain to swell outward to reduce unphysiological, elevated compressive strains. As a first step in a systematic study of this problem, we considered simplified geometric and physical analogs of the problem by studying the bulging of an elastic solid when constrained by a moving boundary with a circular opening. A thorough review of existing geometric models proposed in the medical literature and a study of their explicit relationships between geometric quantities show that these models have little to offer, neither for our understanding of the problem, nor for providing general guidelines for medical treatment as they require the knowledge of both 
the bulging angle and height of the bulge. A judicious choice of these parameters provides an a posteriori justification of surgical practices without much scientific merit.

The mechanical investigation of bulging, however, reveals a number of interesting features. When studied within the framework of small deformations, exact solutions for the bulging of a half-space can be completely solved under the assumption of plane strain, plane stress, and axisymmetry. These solutions follow from classical methods in contact mechanics that are adapted for the problem at hand. Using these methods, the bulging shape is found explicitly in a concise form and was found to match well with finite-element simulations, even for moderate deformations. The study of stress suggests the introduction of damage drops, which are drop-like zones of high and low shear stress with singularities that scale as the inverse square root of the distance from the opening. From the exact solution, we can obtain simple asymptotic estimates for the size, orientation, and shape of these damage drops. As expected, the maximal stretch of vertical material lines is located on the axis of symmetry. The location and magnitude of this maximal stretch is found explicitly and adopts a particularly simple and useful form in the incompressible limit. Interestingly, most of the features uncovered during our analysis-the shape of the bulge, the orientation of the drops, and the singularities of the profile- do not depend on the particular choice of material or boundary conditions. In fact, we expect these solutions to be generic for any type of bulging problem.

Our present analysis is clearly limited by the study of an idealized configuration which disregards the anatomically complex geometry of the brain as well as tissue anisotropy, heterogeneity, and rheology. Nevertheless, it provides a theoretical foundation for the generic problem of bulging and a guide for general geometric configurations. In a companion publication [61], we complement the present theoretical study with an exhaustive computational study of bulging based on an anatomically detailed personalized finite-element model of the skull and brain.

Acknowledgments This work was supported by the Wolfson/Royal Society Merit Award to Alain Goriely and the National Institutes of Health Grant U54GM072970 to Ellen Kuhl. The authors are grateful to Michael Sutcliffe for sharing data and an earlier version of his manuscript.

\section{References}

[1] M. C. Papadopoulos, S. Saadoun, D. K. Binder, G. T. Manley, S. Krishna, and A. S. Verkman. Molecular mechanisms of brain tumor edema. Neuroscience, 129(4):10091018, 2004.

[2] A. Marmarou, R. L. Anderson, J. D. Ward, S. C. Choi, H. F. Young, H. M. Eisenberg, M. A. Foulkes, L. F. Marshall, and J. A. Jane. Impact of ICP instability and hypotension on outcome in patients with severe head trauma instability and hypotension on outcome in patients with severe head trauma. SJ. Neurosurg., 75(1S):S59-S66, 1991.

[3] M. Walberer, N. Ritschel, M. Nedelmann, K. Volk, C. Mueller, M. Tschernatsch, E. Stolz, F. Blaes, G. Bachmann, and T. Gerriets. Aggravation of infarct formation 
by brain swelling in a large territorial stroke: a target for neuroprotection? J. Neurosurg., 109:287-293, 2008.

[4] G. E. Lang, D. Vella, S. L. Waters, and A. Goriely. Propagation of damage in brain tissue: Coupling the mechanics of edema and oxygen delivery. Biomechanics and Modelling in Mechanobiology, 14:1197-1216, 2015.

[5] A. Goriely, M. G. D. Geers, G. A. Holzapfel, J. Jayamohan, A. Jérusalem, S. Sivaloganathan, W. Squier, J. A. W. van Dommelen, S. L. Waters, and E. Kuhl. Mechanics of the brain: perspectives, challenges, and opportunities. Biomech. Model. Mechanobiol., 14:931, 2015.

[6] M. E. Fink. Osmotherapy for intracranial hypertension: mannitol versus hypertonic saline. CONTINUUM: Lifelong Learning in Neurology, 18(3, Critical Care Neurology):640-654, 2012.

[7] P. Grände and B. Romner. Osmotherapy in brain edema: a questionable therapy. $J$ Neurosurg. Anesth., 24(4):407-412, 2012.

[8] A. Raslan and A. Bhardwaj. Medical management of cerebral edema. Neurosurgical focus, 22(5):1-12, 2007.

[9] G. Samandouras. The neurosurgeon's handbook. Oxford University Press, 2010.

[10] J. F. Soustiel, G. E. Sviri, E. Mahamid, V. Shik, S. Abeshaus, and M. Zaaroor. Cerebral blood flow and metabolism following decompressive craniectomy for control of increased intracranial pressure. Neurosurgery, 67(1):65-72, 2010.

[11] R. G. Ellenbogen, S. I. Abdulrauf, and L. N. Sekhar. Principles of neurological surgery. Elsevier Health Sciences, 2012.

[12] A. G. Kolias, P. J. Kirkpatrick, and P. J Hutchinson. Decompressive craniectomy: past, present and future. Nature Reviews Neurology, 9(7):405-415, 2013.

[13] J. Chung, O. Y. Bang, Y. C. Lim, S. K. Park, and Y. S. Shin. Newly suggested surgical method of decompressive craniectomy for patients with middle cerebral artery infarction. The neurologist, 17(1):11-15, 2011.

[14] T Kocher. Die therapie des hirndruckes. Hirnerschütterung, Hirndruck und chirurgische Eingriffe bei Hirnkrankheiten, 3:262-266, 1901.

[15] H. Cushing. The establishment of cerebral hernia as a decompressive measure for inaccessible brain tumors: with the description of intermuscular methods of making the bone defect in temporal and occipital regions. Surg. Gynecol. Obstet., 1:297-314, 1905.

[16] J. Ransohoff, M. V. Benjamin, E. L. Gage Jr, and F. Epstein. Hemicraniectomy in the management of acute subdural hematoma. J. Neurosurg., 34(1):70-76, 1971.

[17] W. K. W. Guerra, M. R. Gaab, H. Dietz, J. U. Mueller, J. Piek, and M. J Fritsch. Surgical decompression for traumatic brain swelling: indications and results. J. Neurosurg., 90(2):187-196, 1999. 
[18] D. J. Cooper, J. V. Rosenfeld, L. Murray, Y. M. Arabi, A. R. Davies, P. D'Urso, T. Kossmann, J. Ponsford, I. Seppelt, P. Reilly, et al. Decompressive craniectomy in diffuse traumatic brain injury. New England J. Med., 364(16):1493-1502, 2011.

[19] F. Servadei. Clinical value of decompressive craniectomy. New England Journal of Medicine, 364(16):1558-1559, 2011.

[20] S. Honeybul, K. M. Ho, and C. R. P. Lind. What can be learned from the DECRA study. World neurosurgery, 79(1):159-161, 2013.

[21] E. Bor-Seng-Shu, E. G. Figueiredo, R. L. O. Amorim, M. J. Teixeira, J. S. Valbuza, M. M. de Oliveira, and R. B. Panerai. Decompressive craniectomy: a meta-analysis of influences on intracranial pressure and cerebral perfusion pressure in the treatment of traumatic brain injury: A review. Journal of neurosurgery, 117(3):589-596, 2012.

[22] J. V Rosenfeld, A. I. Maas, P. Bragge, M. C. Morganti-Kossmann, G. T. Manley, and R. I. L. Gruen. Early management of severe traumatic brain injury. The Lancet, 380(9847):1088-1098, 2012.

[23] C. L. Sedney, T. Julien, J. Manon, and A. Wilson. The effect of craniectomy size on mortality, outcome, and complications after decompressive craniectomy at a rural trauma center. Journal of neurosciences in rural practice, 5(3):212-217, 2014.

[24] R. S. Chung, J. A. Staal, G. H. McCormack, T. C. Dickson, M. A. Cozens, J. A. Chuckowree, M. C. Quilty, and J. C. Vickers. Mild axonal stretch injury in vitro induces a progressive series of neurofilament alterations ultimately leading to delayed axotomy. J. Neurotrauma, 22(10):1081-1091, 2005.

[25] A. C. Bain and D. F. Meaney. Tissue-level thresholds for axonal damage in an experimental model of central nervous system white matter injury. Journal of biomechanical engineering, 122(6):615-622, 2000.

[26] B. Morrison III, H. L Cater, C. C. B. Wang, F. C. Thomas, et al. A tissue level tolerance criterion for living brain developed with an in vitro model of traumatic mechanical loading. Stapp Car Crash Journal, 47:93, 2003.

[27] X. Li, H. von Holst, and S. Kleiven. Decompressive craniectomy causes a significant strain increase in axonal fiber tracts. Journal of clinical neuroscience, 20(4):509-513, 2013.

[28] M. I Miga, K. D. Paulsen, F. E. Kennedy, A. Hartov, and D. W. Roberts. Model-updated image-guided neurosurgery using the finite element method: Incorporation of the falx cerebri. In Medical Image Computing and Computer-Assisted Intervention-MICCAI'99, pages 900-909. Springer, 1999.

[29] A. Wittek, R. Kikinis, S. K. Warfield, and K. Miller. Brain shift computation using a fully nonlinear biomechanical model. In Medical Image Computing and ComputerAssisted Intervention-MICCAI 2005, pages 583-590. Springer, 2005. 
[30] J. Hu, X. Jin, J. B. Lee, L. Zhang, V. Chaudhary, M. Guthikonda, K. H. Yang, and A. I. King. Intraoperative brain shift prediction using a 3D inhomogeneous patient-specific finite element model. J. Neurosurg., 106(1):164-169, 2007.

[31] K. Miller, G. Joldes, L. Lance, and A. Wittek. Total lagrangian explicit dynamics finite element algorithm for computing soft tissue deformation. Communications in numerical methods in engineering, 23(2):121-134, 2007.

[32] A. Goriely, S. Budday, and E. Kuhl. Neuromechanics: From Neurons to Brain. Advances in Applied Mechanics, 48:79-139, 2015.

[33] H. von Holst and S. Kleiven. The non invasive brain injury evaluation, NIBIE-a new image technology for studying the mechanical consequences of traumatic brain injury. In Traumatic Brain Injury. Intech, 2014.

[34] H. von Holst, X. Li, and S Kleiven. Increased strain levels and water content in brain tissue after decompressive craniotomy. Acta neurochirurgica, 154(9):1583-1593, 2012.

[35] H. von Holst and X. Li. Decompressive craniectomy (DC) at the non-injured side of the brain has the potential to improve patient outcome as measured with computational simulation. Acta neurochirurgica, 156(10):1961-1967, 2014.

[36] T. L. Fletcher, A. G. Kolias, P. J. Hutchinson, and M. P. F. Sutcliffe. A new improved method for assessing brain deformation after decompressive craniectomy. PloS one, 9(10):e110408, 2014.

[37] T. L. Fletcher, A. G. Kolias, P. J. A. Hutchinson, and M. P. F. Sutcliffe. Development of a finite element model of decompressive craniectomy. PloS one, 9(7):e102131, 2014.

[38] E. Münch, P. Horn, L. Schürer, A. Piepgras, T. Paul, and P. Schmiedek. Management of severe traumatic brain injury by decompressive craniectomy. Neurosurgery, 47(2):315323,2000 .

[39] C. R. Wirtz, T. Steiner, A. Aschoff, S. Schwab, H. Schnippering, H. H. Steiner, W. Hacke, and S. Kunze. Hemicraniectomy with dural augmentation in medically uncontrollable hemispheric infarction. Neurosurgical Focus, 2(5):E7, 1997.

[40] J. Flechsenhar, J. Woitzik, K. Zweckberger, H. Amiri, W. Hacke, and E. Jüttler. Hemicraniectomy in the management of space-occupying ischemic stroke. Journal of Clinical Neuroscience, 20(1):6-12, 2013.

[41] P. De Bonis, A. Pompucci, A. Mangiola, L. Rigante, and C. Anile. Post-traumatic hydrocephalus after decompressive craniectomy: an underestimated risk factor. Journal of neurotrauma, 27(11):1965-1970, 2010.

[42] S. Wagner, H. Schnippering, A. Aschoff, J. A. Koziol, S. Schwab, and T. Steiner. Suboptimum hemicraniectomy as a cause of additional cerebral lesions in patients with malignant infarction of the middle cerebral artery. J. Neurosurg., 94(5):693-696, 2001.

[43] N. I. Muskhelishvili. Some basic problems of the mathematical theory of elasticity. Springer Science \& Business Media, 1977. 
[44] G. M. L. Gladwell. Contact problems in the classical theory of elasticity. Springer Science \& Business Media, 1980.

[45] K. L. Johnson. Contact Mechanics. Cambridge University Press, 1987.

[46] F. Dostoevsky. Idiot. Russkii Vestnik. 1868.

[47] A. B. Roitman and S. F. Shishkanova. The solution of the annular punch problem with the aid of recursion relations. International Applied Mechanics, 9(7):725-729, 1973.

[48] T. Shibuya, T. Koizumi, and I. Nakahara. An elastic contact problem for a half-space indented by a flat annular rigid stamp. International Journal of Engineering Science, 12(9):759-771, 1974.

[49] M Kumar and Ku Uma Hiremath. Annular punch problem for an elastic layer overlying an elastic foundation. Indian J. pure appl. Math, 13(5):573-580, 1982.

[50] G. M. L. Gladwell and O. P. Gupta. On the approximate solution of elastic contact problems for a circular annulus. J. of Elasticity, 9(4):335-348, 1979.

[51] J. R. Barber. The solution of elasticity problems for the half-space by the method of green and collins. Applied Scientific Research, 40(2):135-157, 1983.

[52] L. A. Mihai and A. Goriely. Positive or negative Poynting effect? the role of adscititious inequalities in hyperelastic materials. Proc. R. Soc. A, 467(2136):3633-3646, 2011.

[53] R. S. Rivlin. Torsion of a rubber cylinder. Journal of Applied Physics, 18(5):444-449, 1947.

[54] M Destrade, MD Gilchrist, JG Murphy, B Rashid, and G Saccomandi. Extreme softness of brain matter in simple shear. International Journal of Non-Linear Mechanics, 75:54$58,2015$.

[55] V. Cerruti. Ricerche intorno all' equilibrio de'corpi elastici isotropi. R. Accad. Lincei Mem. Cl. Sci. Fis. Mat. e Nat., 3(13):81-122, 1882.

[56] A Flamant. Sur la répartition des pressions dans un solide rectangulaire chargé transversalement. CR Acad. Sci. Paris, 114:1465-1468, 1892.

[57] D. J. Unger. Similarity solution of the flamant problem by means of a one-parameter group transformation. Journal of elasticity and the physical science of solids, 66(1):9397, 2002.

[58] W. D. Collins. On the solution of some axisymmetric boundary value problems by means of integral equations. Proceedings of the Edinburgh Mathematical Society (Series 2), 13(03):235-246, 1963.

[59] J. R. Barber. Elasticity. Springer, 1992.

[60] A. E. Green and W. Zerna. Theoretical Elasticity. Dover, New York, 1992.

[61] J. Weickenmeier, C. Butler, P. Young, A. Goriely, and E. Kuhl. The mechanics of decompressive craniectomy: Personalized simulations. Preprint, 2016. 